\title{
On the Optimality of Resetting Executive Stock Options $^{1}$
}

\author{
Viral V. Acharya Kose John Rangarajan K. Sundaram
}

December 8, 1999

\footnotetext{
${ }^{1}$ We are grateful to David Yermack and Lee-Bath Nelson for helpful suggestions; and to an anonymous referee for detailed comments on earlier drafts that led to an extensive revision of the original paper. All three authors are at the Department of Finance, Stern School of Business, New York University, 44 W. 4th Street, New York, NY 10012. Their respective e-mail addresses are vacharya@stern.nyu.edu, kjohn@stern.nyu.edu, and rsundara@stern.nyu.edu.
} 


\begin{abstract}
Recent empirical work has documented the tendency of corporations to reset strike prices on previously-awarded executive stock option grants when declining stock prices have pushed these options out-of-the-money. This practice has been criticised as counter-productive since it weakens incentives present in the original award.

We find that although the anticipation of resetting will typically result in a negative effect on initial incentives, resetting can still be an important, value-enhancing aspect of compensation contracts, even from an ex-ante standpoint. Indeed, we find in a precise sense that some resetting is almost always optimal. We also characterize the conditions that affect the relative optimality of resetting. We find, for example, that the relative advantages of resetting decrease as managerial ability to influence the resetting process increases, as the relative importance of external (industryor economy-wide) factors in return generation increases, and as the direct or indirect costs of replacing the incumbent manager decrease. Our analysis suggests, in summary, that the case against resetting is quite weak.
\end{abstract}




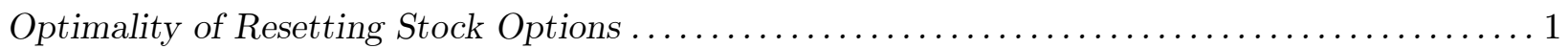

\section{Introduction}

In recent years, stock options have become an important component of overall executive compensation. For such options to serve as a credible incentive device, one might believe that the terms of the original contract should not be amended, especially when declining share prices have pushed the options out of the money. Nonetheless, considerable evidence (see Section 2) suggests that such "resetting" does take place, typically in the form of a reduction in the original exercise price to bring the options closer to the money. Reaction to this practice has been almost uniformly negative. Heavy criticism has come from the financial press ${ }^{1}$ and from large institutional investors such as the State of Wisconsin Investment Board, who suggest that resetting amounts to rewarding management for poor performance, and - more importantly - that it destroys incentives present in the original contract. Weighing in, the U.S. Financial Accounting Standards Board has recently proposed an amendment to current accounting rules that is aimed at making it more expensive for companies to reprice previously-awarded options. ${ }^{2}$

The optimality and incentive effects of resetting have not, thus far, been addressed in an equilibrium contracting model in the theoretical literature on managerial compensation. This is somewhat surprising since resetting has been the theme of a number of recent empirical studies. Our paper is aimed at filling this gap. We develop a model for analysis of this subject, and examine the extent to which the arguments against allowing resetting are justified. Our main conclusion is simply stated: we find that although the anticipation of resetting can have a substantial negative effect on initial incentives, resetting can nonethless be an important, value-enhancing aspect of corporate compensation contracts, even from an ex-ante standpoint. Indeed, we find in our model that some resetting is almost always optimal. Our analysis calls into question the basis for much of the criticism of resetting. We elaborate on these points below. A discussion of the related theoretical and empirical literature follows in Section 2.

Our model builds on the usual agency setting in which an effort-averse manager (the "agent") is provided long-term incentives in the form of call options on the firm's equity by the firm's shareholders (collectively called the "principal"). We consider the following question: suppose an initial compensation contract for the manager is given, and interim information becomes available on the state of the world. From the standpoint of ex-ante value maximization, can it be optimal for the principal to agree to amend the terms of the original option award to reflect this additional information?

A little reflection reveals the trade-off implicit in this question. Credible pre-commitment by the principal to not reset contract terms gives the manager a strong incentive to avoid states from which continuation prospects are poor; the incentive is obviously weaker if the manager anticipates amendment of the contract terms at these states. However, there is a cost to such commitment: the inability to reset the contract in poor states will demotivate the manager at these points,

\footnotetext{
${ }^{1}$ See, e.g., "If the Price isn't Right, Just Change It" by David Johnston, New York Times, July 15, 1998; or "Lowering the Bar" by Joann Lublin, Wall Street Journal, April 8, 1999; among many others.

${ }^{2}$ The amendment proposes that repriced options be classified as variable-strike rather than fixed-strike options. This would require companies to record depth-in-the-money at exercise time as compensation expense, rather than depth-in-the-money at award time. (The latter amount is typically zero since most new options are awarded at-themoney.) For more on the accounting treatment of options, see Opinion No. 25 (1972) of the Accounting Principles Board, and Statement of Financial Accounting Standards No. 123 (1995) of FASB.
} 
leading to poor continuation outcomes for the principal as well. (Indeed, corporations routinely justify resetting as necessary to "reincentivize" employees demotivated by underwater options.) More generally, by allowing dependence on interim information, resetting allows the manager's final payoffs to incorporate a greater degree of sensitivity to the realized path; this flexibility is lost if the principal commits to not reset the contract. Thus, resetting implies two competing effects - a negative "feedback" effect on initial incentives and a positive "incentivization" effect that gives the principal greater ability to fine-tune incentives - and its optimality depends on which effect predominates. ${ }^{3}$

Exploiting this tension, we look for conditions under which resetting could be optimal from an ex-ante standpoint. In Section 4, the first step in our analysis, we compare two natural benchmark strategies for the principal: one, the strategy of pre-commitment, which rules out resetting, and the other, the strategy of reincentivization, which resets at the interim information point whenever superior continuation values result from doing so. Our first result is a particularly unambiguous one: we describe a class of linear models in which we show that it is strictly better from an ex-ante standpoint for the principal to agree to reincentivize the manager fully upon the interim information revelation than to pre-commit to not altering the initial award. We then show that the intuition underlying this construction extends more generally to models in which the manager's cost of effort is small in an appropriate sense; we illustrate this using a quadratic cost structure for effort.

These results on the superiority of reincentivization strategies in some settings are, in themselves, sufficient to refute the criticism of resetting as necessarily suboptimal. Nonetheless, the ambiguity that remains in other cases (with either benchmark strategy dominating the other depending on the specific parameterization) raises the question whether further strengthening of these results is possible. Section 5 answers this question in the affirmative. ${ }^{4}$ The analysis in this section is motivated by the observation that - by resetting whenever it is profitable for the continuationreincentivization strategies may involve "too much" resetting in some circumstances given the feedback effect of resetting. The broader class of strategies we introduce here provides the principal with more flexibility in this direction; of course, the benchmark strategies arise as special cases. We find now that no ambiguity remains: optimal compensation strategies in all cases involve at least some resetting. In particular, (a) where reincentivization strategies were already better than pre-commitment, resetting obviously continues to be optimal, but (b) where pre-commitment was initially superior to reincentivization, using a positive level of resetting, but one smaller than would be used under reincentivization, does better than both.

Building on this foundation, Section 6 then turns to an important concern: the ability of managers to "manipulate" the compensation committee in the resetting decision. Our model enables us to formalize this question in a particularly attractive way. Specifically, we have shown that the optimal level of resetting (which takes into account the feedback effect) typically differs from the level under reincentivization (which looks only at continuation values). This makes the planned resetting vulnerable, at the time of reset, to a "let-bygones-be-bygones" argument from the man-

\footnotetext{
${ }^{3}$ Another side of this trade-off, which highlights initial rather than continuation incentives, was pointed out by this paper's referee. Resetting contract terms in poor states reduces a risk-averse manager's compensating differential for risk-bearing. Ceteris paribus, this reduces compensation expense for the principal. However, the feedback effect of resetting weakens initial incentives, and restoring incentives requires raising compensation levels. The formal analysis in this paper incorporates both aspects of the trade-off.

${ }^{4}$ Sections 5 and 6 were motivated by the referee's comments on an earlier version of our paper.
} 
ager in favor of the efficient continuation that would result under reincentivization. Of course, from the point of view of maintaining credibility in future contracting - whether with the same or other managers - and providing appropriate incentives, it would not be in the principal's interest to give in to this argument. ${ }^{5}$ However, to capture the possibility that the interests of shareholders and the compensation committee are imperfectly aligned, we assume that such managerial intervention is successful a given fraction of the time. Taking this as a parameter, we re-examine optimal reset strategies.

We find that in all cases it still remains optimal to allow for some degree of resetting in the original contract. However, the optimal level of resetting is smaller than if managerial manipulation were not a concern; indeed, it declines monotonically as managerial influence over resetting increases. To understand this, recall that in general the optimal level of resetting is smaller than that under reincentivization. Thus, in the event that managerial intervention is successful, "too much" resetting would result, weakening initial incentives. To compensate for this, the extent of resetting that would otherwise occur must be reduced; however, this reduction never takes on the form of zero resetting.

To complete this analysis, we ask a final question: what if managerial influence can be ruled out in just the one case where the principal commits to no resetting at all, but all positive levels of resetting remain vulnerable to managerial influence? (Although a commitment to no resetting at all also involves inefficient continuations, it appears plausible that it can be credibly enforced by corporations simply adopting this as a rule.) We find even here that in "most" cases, it continues to be optimal to not pre-commit to zero resetting. The exceptions occur when managerial influence is very strong (so the reincentivization continuation occurs with a very high probability regardless of what is planned) and reincentivization outcomes are dominated by those under pre-commitment.

To summarize, the combined results of Sections 4-6 show that the argument against resetting is quite weak. Section 4 shows, first of all, that the no-resetting strategy is dominated in robust classes of problems by the opposite strategy of resetting optimally for the continuation whenever this is profitable. Using a more general class of strategies, Section 5 then shows that it is actually optimal in all cases to use at least some degree of resetting. Section 6 shows that this conclusion remains true even where managers can adversely affect matters by sometimes causing "too much" resetting to occur. Lastly, even where pre-commitment to no resetting can be credibly enforced but any positive level of resetting is vulnerable to managerial manipulation, the former emerges superior only where a specific restrictive combination of conditions happend to hold.

In the final part of the paper, we examine a number of other extensions of the basic model, including two of particular interest. The first concerns settings where either party can sever the relationship when the interim information revelation occurs. (Apart from the need to reincentivize employees, companies frequently justify resetting underwater options as necessary to retain scarce managerial talent.) We find that this causes no change in our conclusion that resetting can be an optimal strategy under general conditions. In particular, we find that the greater the explicit or implicit (i.e., productivity-related) costs of replacing the incumbent manager, the more profitable resetting will be as a compensation strategy.

\footnotetext{
${ }^{5} \mathrm{As}$ is typical in the contracting literature, we view our two-period model as capturing a slice of a more complex pattern of interactions over a longer horizon.
} 
Optimality of Resetting Stock Options ..................................... 4

The second question we examine concerns the impact of the relative control the manager has over the returns distribution on the optimality of resetting. This question has a simple motivation: companies that have repriced options have often sought to justify the action by suggesting that the poor returns preceding the resetting were due more to factors "beyond the manager's control" than a lack of mangerial diligence. We find little support for this argument. Indeed, we find that the less the relative control of the manager over the return distribution, the less optimal resetting becomes. Intuitively, factors that are beyond the manager's direct control but that affect return distributions constitute "background risk" for the manager. It is easier to incentivize the manager when such risk is smaller. Ceteris paribus, this superior ability to fine-tune incentives improves continuation shareholder value; it also provides greater control over the feedback effect. This makes resetting more likely to be ex-ante optimal.

In addition to those mentioned above, some other empirically-relevant features of our paper bear highlighting. First, although our model allows resetting to occur at any point, in equilibrium it only occurs following poor performance. This is consistent with empirical evidence that resetting is a practice invariably preceded by negative shareholder returns (see, e.g., Brenner, Sundaram, and Yermack (1998)). Second, empirically it is known that resetting has a strong inverse relation to firm size, with its incidence increasing sharply as firm size declines (see, e.g., Brenner, Sundaram, and Yermack (1998)). Since it plausibly may be argued that the actions of managers of small firms have greater impact on their return distributions than in large firms, our result that the relative optimality of resetting increases with an increase in managerial control offers one possible explanation of this phenomenon. Third, our model suggests (unintuitively at first blush) that initial option awards will be larger when resetting is possible than when it is ruled out. ${ }^{6}$

This paper is organized as follows. Section 2 describes the related literature. Section 3 presents our model and identifies the benchmark strategies of interest. Section 4 comapres equilibrium outcomes under the benchmark strategies. Section 5 looks at equilibrium under a more general class of strategies. Section 6 considers the impact of managerial influence over resetting. Section 7 looks at other extensions of the model. Section 8 concludes. Proofs of all results omitted in the main body of the paper are provided in the Appendix.

\section{The Related Literature}

Resetting has been the focus of a number of empirical studies. Gilson and Vetsuypens (1993) and Saly (1994) have examined resetting induced by extraordinary circumstances. Gilson and Vetsuypens look at resetting by firms in financial distress during the period 1981-87. Saly focuses on resetting following the stock market crash of 1987.

More recently, Brenner, Sundaram, and Yermack (1998) and Chance, Kumar, and Todd (1997) have each looked at resetting under "normal" circumstances, and characterized the typical reset option. Brenner, Sundaram and Yermack find in their sample that resetting is overwhelmingly preceded by negative stock returns, with its incidence increasing as firm performance worsens; a

\footnotetext{
${ }^{6}$ Since resettable options are worth more than ones that cannot be reset, one might perhaps expect the opposite to be true. However, this ignores the negative feedback effect of resettable options; to overcome this effect and restore initial incentives, it is necessary to increase the number of options.
} 
strong inverse relation remains true even after correcting for industry performance. Resetting is also inversely related to size, being more prevalent among small firms. Concerning the "typical" reset option, they report that in the vast majority of cases in their sample, the new strike price was set to the prevailing stock price at reset time; this resulted, on average, in a $40 \%$ drop in the strike price. Only around half of the reset options also had their maturities extended, with a mean increase of around 30 months. The authors strongly reject the hypothesis that resetting substitutes for other elements of an executive's pay, in particular, for the award of new options.

Chance, Kumar, and Todd study the market, industry, and firm-specific performance for a sample of firms which reset options; their examination covers the period 250 days before and after the resetting event. They also report the presence of a strong inverse relation between resetting and firm performance prior to the reset date. Moreover, they find no significant improvement in shareholder returns after resetting. ${ }^{7}$ Regarding the typical reset option, they find an average $40 \%$ drop in the strike, the same as Brenner, Sundaram, and Yermack. However, in contrast to the latter, they report that fewer than $10 \%$ of reset options in their sample had maturities extended (although the average extension was a high 66 months).

On the theoretical front, Saly (1994) studies a contracting model in which two possible economic environments could prevail, with the abnormal one signifying an economic downturn. The principal and agent agree upon a contract presuming the normal environment will prevail. The state of the world is then revealed (before the agent has taken any payoff-relevant action). If the normal state of the world actually does obtain, the agreed-upon contract is implemented; if not, the contract is reset. Crucially, unlike our model, the interim state in Saly's model is exogenous: it is not related to an earlier action of the agent. Thus, there can be no feedback effect from resetting, so the model cannot be meaningfully used to examine the relative optimality of pre-commitment and reincentivization strategies. Indeed, it is apparent that reincentivization strategies are uniquely optimal in Saly's framework.

Johnson and Tian (1999) study six non-traditional stock option plans including options that can be reset. ${ }^{8}$ They focus on estimating the value of each plan in a Black-Scholes framework; and on gauging its incentive effects by studying its parameter-sensitivities. To this end, they use a risk-neutral valuation setting in which the price process is taken as given and derive closed-form solutions for the options' values. In contrast, we use an optimal contracting model in which the principal's and agent's action choices are explicitly modelled, and the returns process is controlled by the manager, for which he is provided incentives by the principal in the form of options.

Also relevant, but less directly related to our paper, are three other branches of the literature that also look at the feedback effect of recontracting. Two are outgrowths of Hart and Moore (1988), and deal, respectively, with bilateral bargaining (e.g., Aghion, Dewatripont, and Rey (1990), Hart and Tirole (1988)) and debt-recontracting (Aghion and Bolton (1992), Bolton and Scharfstein (1989, 1996), Gale and Hellwig (1989), Hart and Moore (1994, 1995, 1998)). The third concerns design of bankruptcy procedures. The trade-off here is that systems that favor renegotiation in distress

\footnotetext{
${ }^{7}$ Note that, taken by itself, this finding does not contradict the hypothesis that resetting improves employee productivity and firm earnings, since it is not apparent when the anticipation of resetting gets incorporated in stock prices. It is also not clear how returns would have behaved had resetting not taken place, but this counterfactural is, unfortunately, virtually impossible to test.

${ }^{8}$ We thank the referee for drawing this paper to our attention.
} 


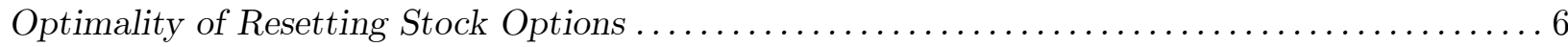

promote efficient continuations in the event of default, but at the cost of encouraging the occurrence of default. One important difference between our paper and those listed here bears mention: moral hazard in our framework arises from the dependence of the firm's cash flow distribution on the effort-averse manager's actions. In much of the recontracting literature, the cash flow process is treated as exogeneous; moral hazard arises from the non-verifiability of the cash flows, that is, from the manager's ability to effect strategic defaults. Thus, the "shirking" or risk-shifting incentives of the renegotiation process are not examined.

\section{The Model}

In this section, we present the structure of our model, identify the benchmark strategies of interest, and define equilibrium in these cases. In Section 4, outcomes under these benchmark strategies are compared. Sections 5-7 then build on the material presented here in several directions of interest.

We consider a two-period model of firm value with dates indexed by $t=0,1,2$. Our model is intended to capture the impact of information revelation in the interim between inception of a compensation contract and its culmination. To this end, we assume that all payoffs in our model are received only at the terminal date $t=2$. This enables the interim date $t=1$ to serve purely as an information event concerning final outcomes that provides a basis for resetting the terms of the initial contract.

The specifics of the model we study are motivated by the popular binomial model of assetpricing theory, augmented to endogenize returns distributions. It will help to refer to Figure 1 while going through the description of this model below.

The firm in our model belongs to an entrepreneur (the "principal") who employs a manager (the "agent") for the two periods. The initial investment in the firm is normalized to unity. There is a single liquidating cash flow from the firm at $t=2$. This cash flow may have three possible values: $H^{2}, H L=L H$, and $L^{2}$, where $H>1>L$. The probabilities of these cash flows depend on the actions (effort levels) taken by the manager in each of the two periods.

The manager's set of possible actions in each period is $A=[0, \bar{a}]$. In the first period, the manager takes an action $a \in A$. Subsequent to this action, a public signal $s \in\{H, L\}$ is observed concerning the terminal cash flows. If the signal $H$ is observed, then terminal cash flows will be either $H^{2}$ or $H L$; if the signal is $L$, then terminal cash flows will be either $L H$ or $L^{2}$. After observing the signal, the manager chooses his second-period action; we will denote by $a_{h}$ the action following the signal $H$, and by $a_{l}$ the action following the signal $L$.

The probabilities of the signals and of the final cash flows depend on the actions taken by the manager. Given the initial period action $a$, the signal $H$ is observed with probability $p(a)$ and the signal $L$ with probability $q(a)=1-p(a)$. If $H$ occurs and the manager follows it up with the action $a_{h}$, then the terminal cash flow $H^{2}$ is realized with probability $p\left(a_{h}\right)$ and the cash flow $H L$ with probability $q\left(a_{h}\right)=1-p\left(a_{h}\right)$. Similarly, if $L$ occurs and the manager follows it with the action $a_{l}$, the cash flows $L H$ and $L^{2}$ are observed with probabilities $p\left(a_{l}\right)$ and $q\left(a_{l}\right)=1-p\left(a_{l}\right)$, respectively. We allow for the possibility that $p(\cdot)$ depends on other "external" parameters as well. We consider such a formulation in Section 7.3; in the interests of notational simplicity, we do not highlight this dependence here. 


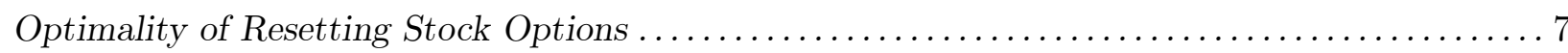

Figure 1: Evolution of Information and Distribution of Terminal Cash Flows

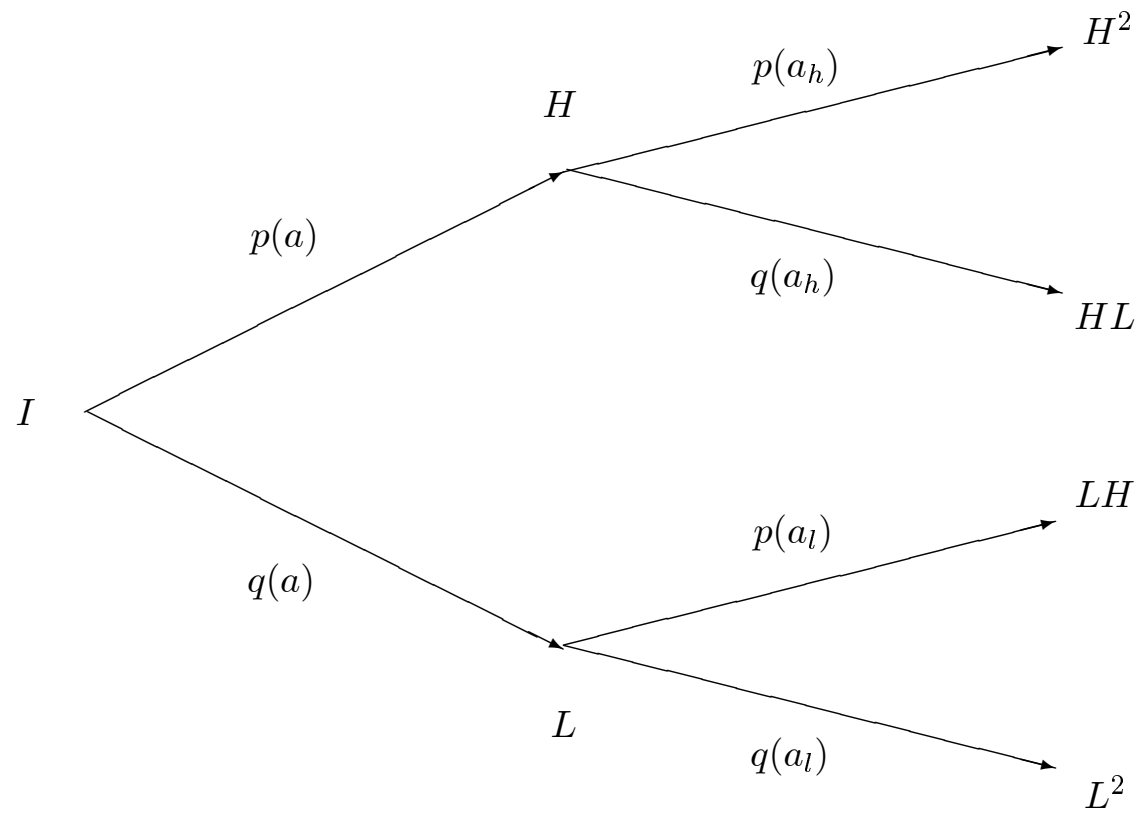




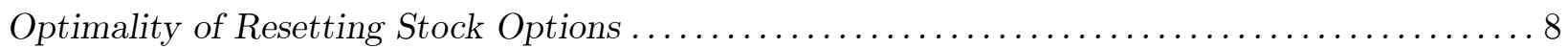

Taking the action $a$ in any period also results in a cost or disutility to the manager of $c(a)$. Let $\mathcal{W}=\left(w_{h h}, w_{h l}, w_{l h}, w_{l l}\right)$ denote a typical compensation profile for the manager, i.e., a typical vector of contingent payoffs at the four terminal nodes. (We will have more to say about the choice of $\mathcal{W}$ shortly.) The manager's objective is to choose an initial action $a$, and contingent continuation actions $a_{h}$ and $a_{l}$ at the nodes $H$ and $L$, respectively, to maximize the discounted expected value of his compensation net of the costs of these actions.

As is usual, we assume the principal is risk-neutral. The principal takes into account the manager's response to any initial offer, and selects a compensation profile that maximizes his own initial expected utility, viz., the discounted expected terminal value of the firm, net of the manager's compensation. We elaborate on these optimization problems below. To keep notation simple, we set all discount rates in the model to zero. We also assume that the principal-manager relationship lasts the full two periods. This is a reasonable assumption in our framework, since ours is essentially a one-period model with an interim information event. Nonethless, in Sections 7.1 and 7.2, we look at the impact of allowing either the principal or the manager to sever the relationship upon revelation of the interim information. We find that while this complicates exposition, under very general and reasonable conditions it does not have an impact on the qualitative nature of our results.

\section{Equilibrium}

We describe the manager's best-response problem first. Let $\mathcal{W}$ be the compensation profile anticipated by the manager. ${ }^{9}$ It is important to note that the anticipated compensation vector need not be the same as that offered by the principal at time $t=0$ if the manager expects that resetting will take place at time $t=1$ (this anticipation of future changes is precisely what causes the feedback effect of resetting). Given $\mathcal{W}$ and any action vector $\mathcal{A}=\left(a, a_{h}, a_{l}\right)$, the manager's continuation utility from $H$, denoted $U_{h}$, is given by

$$
U_{h}=p\left(a_{h}\right) w_{h h}+q\left(a_{h}\right) w_{h l}-c\left(a_{h}\right) .
$$

The manager's continuation utility $U_{l}$ from $L$ is analogously defined. Thus, the manager's initial expected utility from $\mathcal{W}$ and $\mathcal{A}$ is

$$
U\left(a, U_{h}, U_{l}\right)=p(a) U_{h}+q(a) U_{l}-c(a)
$$

It is immediate from these expressions that in response to the anticipated offer $\mathcal{W}$, the manager's optimal actions at the nodes $H$ and $L$, denoted $a_{h}^{*}$ and $a_{l}^{*}$, say, must satisfy:

$$
a_{h}^{*}=\arg \max _{a_{h}} U_{h}\left(a_{h}, w_{h h}, w_{h l}\right) \quad a_{l}^{*}=\arg \max _{a_{l}} U_{l}\left(a_{l}, w_{l h}, w_{l l}\right)
$$

\footnotetext{
${ }^{9}$ At this point, we assume there is no randomization in the resetting process. This is without loss of generality, since our focus in this section and the next (see below) is on two strategies-where contracts are never reset, and where they are reset whenever continuation values can be improved-for which this consideration is not relevant. Section 5 considers a more general class of strategies that includes the possibility of randomization in the resetting decision.
} 
Therefore, letting $U_{h}^{*}=U_{h}\left(a_{h}^{*}, w_{h h}, w_{h l}\right)$ and $U_{l}^{*}=U_{l}\left(a_{l}^{*}, w_{l h}, w_{l l}\right)$, the optimal initial action $a^{*}$ is defined by:

$$
a^{*}=\arg \max _{a} U\left(a, U_{h}^{*}, U_{l}^{*}\right)
$$

Now, given a compensation profile $\mathcal{W}$ and the manager's response $\mathcal{A}=\left(a, a_{h}, a_{l}\right)$ to it, the principal's continuation utility from $H$, denoted $V_{h}$, is given by

$$
V_{h}=p\left(a_{h}\right)\left[H^{2}-w_{h h}\right]+q\left(a_{h}\right)\left[H L-w_{h l}\right]
$$

The principal's continuation utility $V_{l}$ from $L$ is defined analogously. Therefore, the principal's initial expected utility (or value) $V$ is

$$
V=p(a) V_{h}+(1-p(a)) V_{l}
$$

The principal chooses an initial offer $\mathcal{W}$ to maximize $V$ taking into account the manager's reaction to $\mathcal{W}$. In the remainder of this section, we elaborate on this choice through two benchmark cases. The first, that of "pre-commitment," considers the situation where resetting of the initial award is ruled out; the principal makes an initial compensation offer and sticks to it in all circumstances regardless of the interim information revelation. The second case, which we call "contingent resetting" for now (we will provide an alternative label shortly), considers the opposite case where, taking the interim information into account, the contract is reset optimally to maximize the principal's continuation value.

\section{The Compensation Structure}

To keep our focus on the issues motivating this paper, we will center much of the analysis to follow on the situation where the terminal payoffs $\mathcal{W}$ are induced solely by call options on the firm's terminal value. This simplification enables us to sharpen the presentation, and, especially, to capture the tension between the reincentivization and feedback effects of resetting in a transparent form (see the discussion at the end of this section). We emphasize, though, that this assumption is made in the interests of expositional clarity. Our results hold under far more general conditions, as we discuss in Section 7.4. In particular, considerations such as equity or cash bonuses complicate notation, but add little of substance to the analysis.

\section{Equilibrium under Pre-Commitment}

A first case of interest is motivated by a setting where the corporation granting stock options commits to not changing the strike price of the options regardless of what may transpire during the option's life. (This is effectively the behavior corporations have been called upon to adopt by critics of resetting.) In our model, this corresponds to a situation where the principal grants the agent an initial quantity of $\alpha$ call options on the firm's terminal $(t=2)$ value, and commits not to altering the contract at the interim point $t=1$. By analogy with practice, where executive stock 
options are overwhelmingly issued at-the-money, we will assume that any initial options awarded by the principal carry a strike price of unity.

Given any particular value of $\alpha$, the agent's payoffs at maturity under $\alpha$ are easily determined. If, for instance, we have $H L<1$, these terminal payoffs are given by

$$
\begin{array}{lcc}
w_{h h}=\alpha \cdot\left(H^{2}-1\right)^{+}= & \alpha\left(H^{2}-1\right) \\
w_{h l}=\alpha \cdot(H L-1)^{+}= & 0 \\
w_{l h}=\alpha \cdot(L H-1)^{+}= & 0 \\
w_{l l}=\alpha \cdot\left(L^{2}-1\right)^{+}= & 0
\end{array}
$$

Under pre-commitment, the compensation anticipated by the manager at the terminal nodes is the initial one offered by the principal; thus, (3.5) is also the manager's anticipated compensation under $\alpha$. Using this, the manager's response $\mathcal{A}^{*}(\alpha)$ and the equilibrium payoff $V(\alpha)$ to the principal can be computed. The principal then chooses $\alpha$ to maximize $V(\alpha)$.

\section{Equilibrium under Contingent Resetting}

The second benchmark case of interest is where the principal amends the contract at time $t=1$ whenever this improves his continuation value. There are actually two distinct cases to consider here, but only one of real interest, as we discuss below.

Some preliminaries first. In practice, reset options are overwhelmingly reset at-the-money (see, e.g., Brenner, et al [7]). We will assume by analogy that if the initially awarded options in our model are reset at the nodes $H$ or $L$, their strike prices are changed to $H$ or $L$, respectively. Secondly, we also allow resetting to affect the number of options held by the manager. This captures the important possibility that reincentivization can involve more than just a one-for-one exchange of old options for new ones. ${ }^{10}$ Finally, we impose the natural restriction that any offer made by the principal at $t=1$ is firm, that is, it cannot be rescinded or altered at $t=2$ (the problem is trivial otherwise).

A first possibility in defining payoffs under contingent resetting is that the principal retains unlimited right to alter any initial contract terms at $t=1$. In this case, the contract will be reset at each node to maximize the principal's continuation value from that node. Since these revisions will be anticipated by the agent, any initial offer is irrelevant, and the equilibrium is determined by the optimal offers $\alpha_{h}^{*}$ and $\alpha_{l}^{*}$ for the principal at the nodes $H$ and $L$.

The second possibility is motivated by the observation that resetting in practice is almost exclusively associated with underwater options; contract terms are virtually never reset after a good performance when the options are in-the-money, even if managers are "overincentivized" at that point. The analog of this in our model is where the principal retains the right to amend the contract "upwards" in the manager's favor at time $t=1$, i.e., to alter the contract provided the revision also benefits the manager by improving his continuation utility. This leads to an appealing setting in which the principal can reset incentives for demotivated employees by increasing their compensation, but otherwise leaves the contract untouched.

\footnotetext{
${ }^{10}$ For instance, IBM in 1993 offered its managers the opportunity to exchange their previously-issued options for new options with lower strikes at a 5: 2 ratio.
} 
Of these two formulations, the latter is certainly more attractive. It conforms better to observed reality: companies do not, as mentioned, penalize over-incentivized employees by resetting their options downwards following a good performance. It also implicitly suggests that the principal cannot rescind the original contract except at a high cost, which appears reasonable from the standpoint of maintaining credibility in labor markets. ${ }^{11}$ It turns out that the first formulation also can be eliminated on the stronger grounds of analytical irrelevance: it is always dominated (for the principal) by the strategy of only resetting upwards. Indeed, in the latter case, the principal can always make the initial offer of $\alpha=0$ which would permit arbitrary upwards revisions at time $t=1$. Thus, any possible outcome under unrestricted resetting continues to remain feasible. Given this, we focus attention on only the latter case in the rest of this paper, and refer to it as the strategy of reincentivization.

Solving for equilibrium outcomes under reincentivization is a little more complex than under pre-commitment. The procedure works as follows. Given an initial offer of $\alpha$ call options (struck at 1), we first solve for the manager's continuation utilities from $H$ and $L$ under this offer. Then, from each node, we maximize the principal's expected continuation utility over feasible continuation offers (which could involve resetting) taking as given the manager's optimal response to each offer, and subject to the constraint that the new offer must offer the manager at least as much continuation utility as the initial offer. Of course, the principal's and manager's expected utilities in this solution depend on the continuation payoffs associated with the original offer $\alpha$; this provides us an expression for the initial expected utilities in terms of $\alpha$. We then maximize the principal's initial expected utility over all offers $\alpha$ to recover the initial equilibrium offer.

\section{Pre-Commitment vs Reincentivization: The Trade-Offs}

The forces that differentiate reincentivization strategies from pre-commitment can be identified in a particularly transparent form by simply comparing the payoff-generation possibilities when resetting is feasible to those in (3.5) under pre-commitment. So, assume as in (3.5) that $H L<1$ (an identical comparison with the obvious changes, of course, holds if $H L \geq 1$.) Suppose that the principal makes an initial offer of $\alpha$ options, each with a strike price of unity. Suppose further that at the node $L$, these options are replaced by $\beta$ calls each with a strike of $L$. The manager's terminal payoffs are then given by

$$
\begin{array}{cccc}
w_{h h}=\alpha \cdot\left(H^{2}-1\right)^{+} & = & \alpha\left(H^{2}-1\right) \\
w_{h l}=\alpha \cdot(H L-1)^{+} & = & 0 \\
w_{l h}=\beta \cdot(L H-L)^{+} & = & \beta(L H-L) \\
w_{l l}=\beta \cdot\left(L^{2}-L\right)^{+}= & 0
\end{array}
$$

Two effects of resetting are implicit in (3.6). First, we now have the possibility that $w_{l h} \neq w_{h l}$, which was impossible under pre-commitment. More generally, resetting allows for greater sensitivity of final payoffs to the observed history, and thereby expands the set of terminal payoff profiles possible. As a consequence, it allows for superior continuation prospects from $L$ than is possible without resetting. This takes us to the second point, which is that this expansion does not come without

\footnotetext{
${ }^{11}$ Alternatively, it can be interpreted as assuming that the manager also has some bargaining power at reset time, and, in particular, can veto contract changes.
} 
cost. Indeed, in general, resetting at the node $L$ involves improving the manager's continuation prospects also. This reduces the "spread" $\left(U_{h}-U_{l}\right)$ between the manager's continuation utilities from $H$ and $L$, respectively, which then reduces initial incentives to avoid the node $L$. This tension between the positive effect on incentivization and the negative feedback effect on initial incentives determines if resetting is a value-maximizing strategy for the principal. It forms the subject matter of the rest of this paper.

\section{Reincentivization vs Pre-Commitment}

In this section, we present our first results on the optimality of resetting. We compare the two benchmark strategies defined above: pre-commitment strategies, which never reset, and reincentivization strategies, which reset whenever this improves continuation values. We show that under robust conditions, reincentivization strategies can produce superior ex-ante values for the principal despite the negative effect they have on initial incentives. This conclusion is derived in two steps. First, we consider a "linear" family of models and show that for any parameter configuration here, pre-commitment strategies are strictly inferior to reincentivization strategies. Then, we show that the intuition underlying this result extends more generally to situations where the manager's cost of effort is "small" in an appropriately defined sense.

\subsection{A Linear Model}

Intuitively speaking, for reincentivization to dominate pre-commitment, we need a situation where the feedback effect of resetting is minimal, but where the effect of resetting on continuation payoffs is strong. Thus, we must identify a setting in which the manager's continuation utilities from resetting and not resetting do not differ widely (this will minimize the feedback effect); but where the manager's actions in the two cases differ substantially (so the principal gets the benefits of resetting). We describe in this subsection a model with linear payoffs for both players that meets these conditions.

We take the space of feasible actions for the manager to be $[0, \bar{a}]$ for some $\bar{a}<1$. The functions $p(\cdot)$ and $c(\cdot)$ for the manager are both assumed to have linear forms: $p(a)=a$, and $c(a)=k a$, where $k>0$. Further, we will assume that $k<L H-L^{2}$. (Without this restriction, the continuation from $L$ becomes trivial since it will be unprofitable for the principal to ever offer a positive payoff to the manager.) Finally, in the interests of analytical simplicity, we will assume that $H L<1$; this simplifies payoffs in the analysis. ${ }^{12}$

\footnotetext{
${ }^{12}$ One additional point is important. A formal model of the sort described here cannot distinguish between new issues of options and resetting of existing options. It is therefore implicit in the remainder of this paper that no new options are issued at time $t=1$. This is consistent with the finding in Brenner, Sundaram, and Yermack (1998) that resetting does not appear to be a substitute for the granting of new options.
} 


\subsubsection{Equilibrium under Pre-Commitment}

Let $\alpha$ be given. Then, the manager's terminal payoffs at the four nodes are given by (3.5). Therefore, at the node $H$, the manager's action $a_{h}$ is determined as the solution to

$$
\max _{a \in[0, \bar{a}]}\left[a \cdot \alpha\left(H^{2}-1\right)+(1-a) \cdot 0-k a\right]
$$

It is immediate that $a_{h}$ and the manager's continuation utility $U_{h}$ from $H$ are given by

$$
\begin{aligned}
& a_{h}= \begin{cases}0, & \text { if } \alpha\left(H^{2}-1\right)<k \\
\bar{a}, & \text { otherwise }\end{cases} \\
& U_{h}= \begin{cases}0, & \text { if } \alpha\left(H^{2}-1\right)<k \\
\bar{a} \alpha\left(H^{2}-1\right)-k \bar{a}, & \text { otherwise }\end{cases}
\end{aligned}
$$

At $L$, regardless of the choice of $\alpha$, the continuation payoffs for the manager are always zero. It follows trivially that $a_{l}$ and $U_{l}$ are given by

$$
a_{l}=U_{l}=0
$$

Finally, at the initial node, the manager solves $\max _{a \in[0, \bar{a}]}\left\{a U_{h}+(1-a) U_{l}-k a\right\}$. The optimal time -0 action, therefore, is

$$
a= \begin{cases}0, & \text { if } U_{h}-U_{l}<k \\ \bar{a}, & \text { otherwise }\end{cases}
$$

Expressions (4.1)-(4.4) make clear the choices facing the principal. If the principal sets $\alpha$ so that $\left(U_{h}-U_{l}\right)<k$, then from (4.4), the node $H$ is never reached, so from (4.3), the final outcome is $L^{2}$ with certainty. Thus, contingent on this case, the principal's best choice is to set $\alpha=0$, which provides an initial expected utility to the principal of $L^{2}$.

On the other hand, suppose the principal sets $\alpha$ so that $U_{h}-U_{l} \geq k$. Since $k>0$ and $U_{l}=0$, this can happen only if $U_{h}>0$, so $U_{h}$ must be given by the second value in (4.2), i.e., $U_{h}=\bar{a} \alpha\left(H^{2}-1\right)-k \bar{a}$. Therefore, $\left(U_{h}-U_{l}\right) \geq k$ holds if and only if

$$
\alpha \geq \frac{k(1+\bar{a})}{\bar{a}\left(H^{2}-1\right)}
$$

In addition, (4.2) implies that for $U_{h}$ to be given by $\bar{a} \alpha\left(H^{2}-1\right)-k \bar{a}, \alpha$ must also satisfy $\alpha\left(H^{2}-1\right) \geq$ $k$. Now, it is evidently in the principal's interest to have $\alpha$ as small as possible. An easy calculation shows that the least value of $\alpha$ that satisfies both of the required inequalities is when equality holds in (4.5). For this value of $\alpha$, a further computation shows that the principal's initial expected utility is given by $\left[\bar{a}^{2} H^{2}+\bar{a}(1-\bar{a}) H L+(1-\bar{a}) L^{2}-k \bar{a}(1+\bar{a})\right]$. This is larger than $L^{2}$ if, and 
only if, $(\bar{a} H+L)(H-L) \geq k(1+\bar{a})$. But this last inequality always holds, since $k<H L-L^{2}$ by assumption, and $H>L$.

To sum up, therefore, the equilibrium under pre-commitment has the following values for $\alpha$ and the principal's initial expected utility $V$ :

$$
\begin{aligned}
& \alpha=\frac{k(1+\bar{a})}{\bar{a}\left(H^{2}-1\right)} . \\
& V=\bar{a}^{2} H^{2}+\bar{a}(1-\bar{a}) H L+(1-\bar{a}) L^{2}-k \bar{a}(1+\bar{a}) .
\end{aligned}
$$

In this equilibrium, the manager takes the action $\bar{a}$ at the initial node and at the node $H$, but continues with $a_{l}=0$ if the node $L$ is reached.

\subsubsection{The Superiority of Reincentivization}

Under pre-commitment, the continuation value from $L$ was $L^{2}$ for the principal and zero for the manager. If resetting is possible, however, the principal will be able to induce a better action for the manager. Specifically, note that if the principal resets by offering $\beta$ new at-the-money options, the manager's optimal action $a_{l}$ in the continuation from $L$ is

$$
a_{l}= \begin{cases}0, & \text { if } \beta(L H-L)<k \\ \bar{a}, & \text { otherwise }\end{cases}
$$

It is evident that the optimal reset contract is either $\beta=0$ or $\beta=k /(L H-L)$. If $\beta=0$ (i.e., the principal does not reset), then the principal's continuation utility from $L$ is, of course, $V_{l}=L^{2}$. On the other hand, if $\beta=k /(L H-L)$, we have $a_{l}=\bar{a}$, so the principal's continuation utility is

$$
V_{l}=\bar{a} L H+(1-\bar{a}) L^{2}-k \bar{a} .
$$

This quantity is greater than $L^{2}$ under our assumption that $L H-L^{2}>k$. Thus, it is optimal for the principal to reset at $L$ (in particular, by setting $\beta=k /(L H-L)$ ), and this results in the continuation value (4.8) for the principal. The continuation value $U_{l}$ for the manager is

$$
U_{l}=\bar{a} \beta(L H-L)-k \bar{a}=0 .
$$

Observe the important point that the manager's continuation utility from $L$ in this case is the same as without resetting. This means that optimal resetting at $L$ has no feedback effect on the manager's first-period action.

Now, we have seen that the optimal initial offer when there is no resetting is given by

$$
\alpha=\frac{k(1+\bar{a})}{\bar{a}\left(H^{2}-1\right)}
$$


Suppose the principal were to make the initial offer (4.10) when resetting is permitted. We will show that the principal's time- 0 expected utility from making this initial offer is strictly larger than his time 0 expected utility in the pre-commitment equilibrium. Since (4.10) is a feasible, but not necessarily optimal, initial offer in this case, the superiority of reincentivization over precommitment in this setting is proved.

It is easy to check that if the principal makes the initial offer $\alpha$ as defined by (4.10), it is optimal for the principal to never reset contingent on reaching $H$, but to reset to $\beta=k /(L H-L)$ options with a strike of $L$ if the node $L$ is reached. As a consequence:

1. The principal's continuation utility $V_{h}$ from $H$ under this strategy is the same as in the pre-commitment equilibrium.

2. The principal's continuation utility $V_{l}$ from $L$ (given by (4.8)) is strictly higher than the continuation level of $L^{2}$ in the pre-commitment equilibrium.

3. Resetting at $L$ does not change the manager's continuation utility $U_{l}$ from $L$ (see (4.9)), so the manager's first-period incentives are unaffected, and his first-period action continues to be given by $\bar{a}$.

It is immediate from this that the principal's time- 0 value under reincentivization from the feasible initial offer $\alpha$ is strictly larger than that in the pre-commitment equilibrium.

\subsection{The Linear/Quadratic Model}

The driving force behind the unambiguous superiority of reincentivization strategies in the linear model is the ability to reset contracts in the continuation without a feedback effect on initial incentives. A natural question here is: what if the model is such that resetting implies a non-zero feedback effect? In such situations, it would also be necessary to raise the manager's incentives in the initial period to counteract this negative effect; more specifically, the initial offer $\alpha$ would have to be increased to enlarge the utility spread $\left(U_{h}-U_{l}\right)$. Evidently, whether this is profitable for the principal depends on the required increase in initial incentives: if the "bang per buck" is large (a moderate increase suffices to create a substantial effect on the manager's actions), we would expect to be able to profitably implement a change in the initial incentives, and resetting would dominate pre-commitment. Conversely, if a substantial increase is needed, pre-commitment would emerge superior.

Of course, the impact of a change in the utility spread on initial actions depends ultimately on the manager's cost function. Thus, when costs are "small" relative to their productivity impact, we would expect resetting to be superior. In this subsection, we demonstrate this point in the context of a linear-quadratic model, though, of course, the intuition holds more generally. Specifically, we take the functions $p(\cdot)$ and $c(\cdot)$ to be given by

$$
\begin{aligned}
& p(a)=a, \\
& c(a)=\frac{1}{2} k a^{2}, \quad k>0 .
\end{aligned}
$$


For analytical simplicity, we also continue to assume that $H L \leq 1 .{ }^{13}$ Under these parametrizations, Appendix A describes in detail the derivation of equilibrium under both pre-commitment (Appendix A.1) and reincentivization (Appendix A.2).

Based on the discussion above, we would expect that if the parameter $k$ of the cost function is small, it should be possible to make up for the feedback effect, since a small increase in the continuation utility at $H$ will then have a large impact on the initial action. Thus, resetting should emerge dominant in this case. As $k$ increases, ever-larger payouts are required to make up for the feedback effect, and we would expect pre-commitment to fare better.

That this intuition is correct is borne out in Table 1. The table summarizes equilibrium payoffs under either regime for specified parameter values. Three values of $k$ are considered, chosen to highlight the importance of this parameter in the choice between pre-commitment and resetting. For this range of parameter values, equilibrium values and option awards have the following form ( $C$ and $R$ denote pre-commitment and resetting, respectively):

$$
\begin{aligned}
& V_{C}=L^{2}+\bar{a}\left[H L-L^{2}+\bar{a}\left(H^{2}-H L\right)-k \bar{a}-k \bar{a}^{2} / 2\right] . \\
& V_{R}=L^{2}+2 \bar{a}\left[H L-L^{2}-\bar{a} k\right]+\bar{a}^{2}(H-L)^{2} . \\
& \alpha_{C}=k(2+\bar{a}) /\left[2\left(H^{2}-1\right)\right] . \\
& \alpha_{R}=k(1+\bar{a}) /\left(H^{2}-1\right) \quad \beta_{R}=(\bar{a} k) /(H L-L)
\end{aligned}
$$

Several aspects of the table bear highlighting. First, we find (Appendix A.2) that even when resetting is permitted, it is never optimal to reset at the node $H$. Accordingly, Table 1 provides only one reset entry - $\beta$ - which is the number of options in the optimal reset at $L$. Observe that since $\beta$ is strictly positive for the parameter values in the table, resetting is strictly preferable to not resetting in the continuation from $L$.

Secondly, the size of the initial offer $\alpha$ is substantially higher in each case under reincentivization than under pre-commitment. This is expected: resetting at $L$ has an unfavorable feedback effect on initial incentives for the manager, since it lowers the utility spread $\left(U_{h}-U_{l}\right)$. The higher $\alpha$ is required to compensate for this.

Thirdly, as expected, reincentivization emerges superior to pre-commitment at low values of $k$ : it is possible in these cases to use the higher value of $\alpha$ to compensate for the feedback effect profitably. (See, e.g., the equilibrium outcomes corresponding to $k=0.050$ in the table.) As $k$ increases, the gap between the regimes narrows, and the two are roughly equal from the principal's standpoint at the table's middle value of $k=0.075$. At still higher values of $k$, pre-commitment becomes uniquely dominant. ${ }^{14}$

Finally, note that the manager is always better off under a reincentivization regime than a precommitment regime. This improvement obtains from two sources. On the one hand, the resettable

\footnotetext{
${ }^{13}$ This assumption is not central to the results that follow. Qualitatively identical behavior emerges when $H L>1$. However, closed-form solutions appear hard to obtain in this case, since the algebra becomes dense.

${ }^{14}$ More precisely, using (4.13)-(4.14), the cross-over point is seen to be $k=0.062$ (approx).
} 


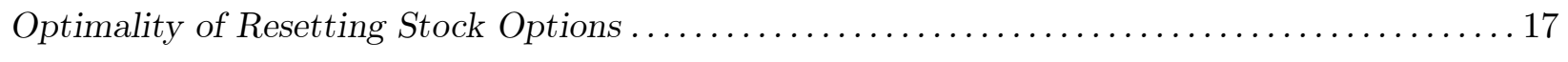

Table 1: Pre-commitment vs Resetting in the Linear/Quadratic Model

This table describes the structure of equilibrium payoffs and actions under both pre-commitment and resetting in the linear/quadratic model of Section 4.2. The base parameters are fixed at $H=1.20$, $L=0.833$, and $\bar{a}=0.90$. Three values are considered for the quadratic cost parameter $k$. The remaining notation in the table is taken from the text: (i) $V$ and $U$ are, respectively, the principal's and agent's expected utilities in equilibrium, and (ii) $\alpha$ is the number of at-the-money options in the initial offer, and (iii) $\beta$ is the number of at-the-money options offered under the reincentivization strategy when resetting at $L$.

\begin{tabular}{||l|c|c|c|c||}
\hline \multicolumn{5}{|c||}{$k=0.050$} \\
\hline Regime & $V$ & $U$ & $\alpha$ & $\beta$ \\
\hline Pre-commitment & 1.2671 & 0.0203 & 0.165 & n.a. \\
\hline Reincentivization & 1.2723 & 0.0405 & 0.216 & 0.270 \\
\hline
\end{tabular}

\begin{tabular}{||l|c|c|c|c||}
\hline \multicolumn{5}{|c||}{$k=0.075$} \\
\hline Regime & $V$ & $U$ & $\alpha$ & $\beta$ \\
\hline Pre-commitment & 1.2378 & 0.0304 & 0.247 & n.a. \\
\hline Reincentivization & 1.2318 & 0.0608 & 0.324 & 0.405 \\
\hline
\end{tabular}

\begin{tabular}{||l|c|c|c|c||}
\hline \multicolumn{5}{|c||}{$k=0.100$} \\
\hline Regime & $V$ & $U$ & $\alpha$ & $\beta$ \\
\hline Pre-commitment & 1.2084 & 0.0405 & 0.330 & n.a. \\
\hline Reincentivization & 1.1913 & 0.0810 & 0.432 & 0.540 \\
\hline
\end{tabular}

options the manager initially receives under reincentivization are each worth more, a priori, than the fixed options he receives under pre-commitment. Compounding this effect, he also receives more options initially under reincentivization than under pre-commitment. ${ }^{15}$

\section{$5 \quad$ Resetting in a More General Setting}

By resetting optimally whenever continuation values are increased by so doing, it appears likely that reincentivization strategies will involve "too much" resetting in some circumstances given the feedback effect on initial incentives. It is particularly noteworthy then that despite this limitation, reincentivization can be strictly value-improving over pre-commitment. Nonetheless, it is of interest to inquire whether the results of the previous section can be further strengthened by improving the principal's flexibility in the resetting decision. In this section, we examine equilibrium outcomes under such a broader class of strategies. For specificity, we continue with the linear-quadratic model of the previous subsection.

\footnotetext{
${ }^{15}$ The referee pointed out that this limited observation should not be taken to imply strict improvement in the manager's welfare occurs whenever resetting is "legally" permissible. Specifically, consider the "supergame" in which the principal first chooses between reincentivization and pre-commitment, and then, given the regime chosen, the game evolves as described here. In this supergame, Table 1 shows that for some parametrizations, the principal will find it optimal to pre-commit to no resetting. Thus, allowing the principal this initial choice leaves the manager with the same welfare as when resetting is prohibited altogether.
} 
We expand the set of compensation strategies in two directions. First, unlike under reincentivization, we do not insist that resetting involve choosing the level that maximizes the principal's continuation value; instead we allow the principal to commit to any particular choice $\beta$ of reset options at the node $L$. (Since resetting at $H$ never occurs in equilibrium, we avoid spurious generality in the notation, and focus only on resetting at $L$.) Second, we permit randomization in the resetting decision; that is, rather than require that contracts always be reset when this is profitable for the continuation (as under reincentivization) or never reset in any circumstances (as under pre-commitment), we allow the principal to choose a probability $\pi$ that the contract will be reset.

Thus, the principal's strategy now involves the choice of three quantities: the size $\alpha$ of the initial award, the probability $\pi$ of resetting at $L$, and the size $\beta$ of the reset award. Of course, the benchmark strategies are now special cases. For example, if $\pi=0$ or $\beta=0$, there is no resetting at $L$; this corresponds to the case of pre-commitment. Similarly, the strategy of reincentivization obtains if $\pi=1$ and $\beta$ is set equal to the level $\beta^{*}$ that would prevail in this case. Moreover, there are now two ways of controlling the feedback effect: by reducing the size of the reset award $\beta$, or by reducing the probability of resetting $\pi$.

Given $(\alpha, \pi, \beta)$, the manager receives the terminal payoffs (3.5) if resetting does not occur at $L$, and the terminal payoffs (3.6) if it does. Using these payoffs, we can easily compute:

1. The manager's optimal action $a_{h}$ at $H$, and the resulting continuation values $U_{h}$ and $V_{h}$ for the manager and principal.

2. Conditional on no resetting at $L$, the manager's optimal action $a_{l}^{n}$ at $L$ and the resulting continuation values $U_{l}^{n}$ and $V_{l}^{n}$ for the manager and principal.

3. Conditional on resetting occurring at $L$, the optimal action $a_{l}^{r}$ at $L$, and the resulting continuation values $U_{l}^{r}$ and $V_{l}^{r}$ for the manager and principal.

The manager now picks his initial action $a$ to maximize his time -0 expected utility:

$$
a U_{h}+(1-a)\left[\pi U_{l}^{r}+(1-\pi) U_{l}^{n}\right]-c(a)
$$

The optimal action $a^{*}$ then determines the principal's expected value as

$$
a^{*} V_{h}+\left(1-a^{*}\right)\left[\pi V_{l}^{r}+(1-\pi) V_{l}^{n}\right]
$$

The principal now picks $\alpha, \pi$, and $\beta$ to maximize (5.18). We will refer to the value of $\beta$ in the optimal contract as the optimal reset level.

Table 2 presents equilibrium outcomes under optimal resetting for the same sets of parameter values as in Table 1. (To keep the notation distinct, a "hat" is used over the relevant variables here.) A comparison of the tables reveals several interesting points.

First, we mentioned at the top of this section that reincentivization could potentially involve "too much" resetting. This is borne out by a comparison of the numbers in Tables 1 and 2. For example, at $k=0.100$, we have $\beta=0.540$ under reincentivization; however, Table 2 shows that the optimal reset level in this case is just $\hat{\beta}=0.250$. 


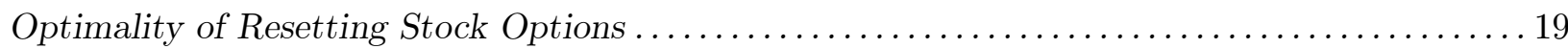

Table 2: Optimal Resetting in the Linear/Quadratic Model

This table describes equilibrium values of payoffs and actions in the linear/quadratic model for the general class of strategies defined in Section 5. The parameter values are identical to those used in Table 1. Of the notation in the table: (i) $k$ is the quadratic cost parameter in (4.12); (ii) $\hat{V}$ and $\hat{U}$ are, respectively, the principal's and agent's expected utilities in equilibrium, (iii) $\hat{\alpha}$ is the number of options in the initial award, (iv) $\hat{\beta}$ is the number of options in the optimal reset at $L$, and (v) $\pi$ is the equilibrium probability of resetting at $L$.

\begin{tabular}{||c||c|c|c|c|c||}
\hline$k$ & $\hat{V}$ & $\hat{U}$ & $\hat{\alpha}$ & $\hat{\beta}$ & $\pi$ \\
\hline 0.025 & 1.3145 & 0.0207 & 0.109 & 0.138 & 1.00 \\
\hline 0.050 & 1.2775 & 0.0316 & 0.194 & 0.202 & 1.00 \\
\hline 0.075 & 1.2446 & 0.0394 & 0.270 & 0.221 & 1.00 \\
\hline 0.100 & 1.2133 & 0.0492 & 0.351 & 0.250 & 1.00 \\
\hline 0.125 & 1.1829 & 0.0582 & 0.431 & 0.260 & 1.00 \\
\hline
\end{tabular}

Second, Table 2 shows that it is optimal in all cases to have some degree of resetting; indeed, the optimal level of resetting in all cases lies strictly between $\beta=0$ (the resetting level under precommitment) and $\beta=\beta^{*}$ (the resetting level under reincentivization). This shows, in particular, that the inferiority of reincentivization compared to pre-commitment at high values of $k$ stems from "excessive" resetting.

Thirdly, the optimal reset level $\hat{\beta}$ is increasing in $k$. This suggests that resetting is likely to remain optimal at all levels of the cost parameter $k$ (it is already optimal for all small $k$ because even reincentivization does better than pre-commitment in this case).

Lastly, allowing for randomization does not appear to have any impact on the equilibrium. In all cases in Table 2 (as also in a wide variety of other parameterizations we tried), the equilibrium value of $\pi$ is unity. This appears to arise from the feature that $\beta$ and $\pi$ are partially substitutes: a lowering of either reduces feedback effects (and expected continuation values). Moreover, even with $\pi$ fixed at unity, the continuum between pre-commitment and reincentivization is achieved by letting $\beta$ vary between 0 (the resetting level under pre-commitment) and $\beta^{*}$ (the resetting level under reincentivization). In the remainder of the paper, therefore, we omit this parameter in the interests of notational simplicity.

\section{$6 \quad$ The Impact of Mangerial Influence over Resetting}

An important issue concerning resetting in general is the influence managers may have over the resetting decision and the impact of this on the optimality of allowing for resetting. ${ }^{16}$ The model we have developed here enables a sharp formalization and study of this question.

Specifically, the results of the last section indicate that resetting is clearly an optimal policy to follow in general even from the point of view of maximizing ex-ante shareholder value. Yet, the nature of the optimal reset level raises questions of enforceability. Unlike the reincentivization

\footnotetext{
${ }^{16}$ This issue was raised by the referee in a report on an earlier version of this paper.
} 
policy, which is based solely on choosing an efficient continuation, the optimal reset policy also takes into account the feedback efect in choosing the level of resetting to follow; thus, it is not, in general, efficient for the continuation alone. This makes the policy vulnerable at the time of reset to an argument from the manager in favor of resetting efficiently for the continuation alone regardless of what was envisaged in the original strategy. In this section, we examine how the equilibrium is affected if the manager has at least some ability to influence the resetting decision in this way.

A parenthetic discussion is important here regarding two questions: (i) Why would the principal wish to stick to the level of resetting envisaged in the original contract? and (ii) Given this, why might managerial influence succeed in changing this decision? Concerning the first question: as is typical in the contracting literature, we view our model as a simple way of capturing a more complex interaction over a longer horizon. From the point of view of retaining credibility in future contracting - with the same or other managers - and providing appropriate incentives, it is then important for the principal to stick to the resetting originally envisaged. On the other hand, in allowing managerial influence over the resetting decision to sometimes be successful, we aim to capture the important possibility that shareholder interests are not perfectly aligned with those of the compensation committee.

In notational terms, we extend the model of the previous section as follows. Let $(\alpha, \beta)$ be any contract chosen by the principal, and let $\beta^{*}$ denote the optimal level of resetting for the continuation alone, i.e., the level of resetting that would occur under reincentivization. We formalize successful managerial influence over the resetting decision with a parameter $e \in[0,1]$ by assuming that with probability $e$, the manager is successful in having the contract reset to the efficentcontinuation contract $\beta^{*}$ at the node $L$; while, with probability $1-e$, the originally envisaged reset $\beta$ is implemented at $L$. With the parameter $e$ taken as exogeneously specified, we examine the question: how does this change the optimality of allowing for resetting? In particular, what optimal level of resetting $\beta$ will be envisaged in the original contract if the ability of managers to possibly have the contract reset to $\beta^{*}$ (with probability $e$ ) is anticipated?

Intuitively, the direction of the impact is not hard to see. The level $\beta^{*}$ involves more resetting than is optimal given the feedback effect. To counteract this effect, the resetting level $\hat{\beta}$ envisaged in the original contract must be reduced. In particular, we would expect the optimal level of planned resetting (denoted, say, $\hat{\beta}_{e}$ ) to decline monotonically in $e$. However, since $\hat{\beta}_{e}$ cannot become negative, it is interesting to ask whether $\hat{\beta}_{e}$ becomes zero for high $e$; and whether resetting becomes suboptimal as managerial influence over the resetting decision increases.

Table 3 provides the answer to this question in the context of the linear-quadratic model developed earlier. (In the interests of brevity, we do not go into the details of the derivation of equilibrium here; it is apparent how the presentation of the previous sections may be extended towards this end.) To retain comparability, the values of all parameters (except for $e$ ) are kept the same as in the previous tables. In particular, the resetting level $\beta^{*}$ under reincentivization can be read off Table 1 ; and the optimal resetting level $\hat{\beta}$ in the absence of managerial influence can be read off Table 2. The subscript $e$ is added to all equilibrium quantities to underline their dependence on this parameter.

The table confirms the intuitive arguments made above, and also establishes that planning for a positive level of resetting remains optimal even when managerial influence becomes "large." As anticipated, the optimal reset level $\hat{\beta}_{e}$ envisaged in the optimal contract decreases as $e$ increases. 
Table 3: Optimal Resetting and Mangerial Influence

This table describes equilibrium payoffs and contracts in the linear/quadratic model extended to the setting of Section 6 . The base parameter values are fixed as in Tables 1 and 2. The parameter $e \in[0,1]$ denotes managerial influence over the resetting decision as defined in Section $6 ; e=0$ corresponds to the case of no influence. $\beta^{*}$ is the reset award that results from successful managerial intervention in the resetting decision. Given $e$, (i) $\hat{V}_{e}$ and $\hat{U}_{e}$ are, respectively, the principal's and manager's expected utilities in equilibrium, (ii) $\hat{\alpha}_{e}$ is the initial option award, and (iii) $\hat{\beta}_{e}$ is the reset award envisaged in the original contract.

\begin{tabular}{||c|c|c|c|c|c||}
\hline \multicolumn{7}{||c||}{$k=0.050$} \\
\hline$e$ & $\hat{V}_{e}$ & $\hat{U}_{e}$ & $\hat{\alpha}_{e}$ & $\hat{\beta}_{e}$ & $\beta^{*}$ \\
\hline 0.1 & 1.2769 & 0.03213 & 0.195 & 0.199 & 0.270 \\
0.3 & 1.2759 & 0.03390 & 0.199 & 0.197 & 0.270 \\
0.5 & 1.2749 & 0.03529 & 0.203 & 0.192 & 0.270 \\
\hline
\end{tabular}

\begin{tabular}{||c|c|c|c|c|c||}
\hline \multicolumn{7}{||c||}{$k=0.075$} \\
\hline$e$ & $\hat{V}_{e}$ & $\hat{U}_{e}$ & $\hat{\alpha}_{e}$ & $\hat{\beta}_{e}$ & $\beta^{*}$ \\
\hline 0.1 & 1.2434 & 0.04113 & 0.274 & 0.215 & 0.405 \\
0.3 & 1.2408 & 0.04539 & 0.285 & 0.213 & 0.405 \\
0.5 & 1.2383 & 0.04972 & 0.296 & 0.212 & 0.405 \\
\hline
\end{tabular}

\begin{tabular}{||c|c|c|c|c|c||}
\hline \multicolumn{7}{||c||}{$k=0.100$} \\
\hline$e$ & $\hat{V}_{e}$ & $\hat{U}_{e}$ & $\hat{\alpha}_{e}$ & $\hat{\beta}_{e}$ & $\beta^{*}$ \\
\hline 0.1 & 1.2112 & 0.05160 & 0.358 & 0.237 & 0.540 \\
0.3 & 1.2068 & 0.05787 & 0.373 & 0.232 & 0.540 \\
0.5 & 1.2024 & 0.06443 & 0.390 & 0.230 & 0.540 \\
\hline
\end{tabular}


In particular, $\hat{\beta}_{e}$ is everywhere smaller that the optimal reset level $\hat{\beta}$ in the absence of managerial influence. Second, as $e$ increases, the manager's utility level in equilibrium increases, while the principal's falls. This is intuitive, since $e$ measures the relative power of the manager in one aspect of the interaction. Third, the rate of decline of $\hat{\beta}_{e}$ as $e$ increases is very slow: even at very high values of $e, \hat{\beta}_{e}$ is strictly positive. In words, planning for some positive level of resetting remains optimal even when managerial influence over resetting becomes large. ${ }^{17}$ Lastly, as with $\hat{\beta}$ in Table 2 , for any fixed $e$, the optimal level $\hat{\beta}_{e}$ increases in $k$. Thus, optimal resetting remains strictly positive at all levels of $k$.

One final point is important. In computing these equilibrium values, we have assumed that whenever the principal commits to an inefficient continuation, the manager can argue successfully (with probability $e$ ) in favor of the efficient continuation $\beta^{*}$. An exception, however, could arise where the principal pre-commits to no resetting. In this case, inefficient continuations will certainly result from some states. Nonetheless, it appears plausible that by the corporation accepting no resetting as a general policy, adverse managerial influence at these nodes can be forestalled.

Accepting this possibility for the moment, how would the optimality of resetting change? As $e$ increases towards its upper bound of unity, the likelihood of resetting according to $\beta^{*}$ increases towards unity, so the principal's value in equilibrium approaches the value under reincentivization. Where reincentivization is anyway superior to pre-commitment (e.g., $k=0.050$ ), this makes no difference to the optimality of resetting. However, where pre-commitment is preferable to reincentivization, this means that as managerial influence over resetting becomes high, it could become preferable to pre-commit to no resetting at all.

Two caveats are, however, important here. First, note from Table 3 that managerial influence has to be quite high before pre-commitment becomes optimal. For example, in the middle panel $(k=0.075)$, pre-commitment dominates reincentivization, but even at $e=0.50$, optimal resetting is preferable to pre-commitment. Second, it is very important that the commitment to no resetting be credible, i.e., that managerial attempts to have the contract reset not work. If such credible commitment is doubtful or impossible, then the structure of the optimal policy is as outlined in Table 3, and this always involves some resetting.

\section{Extensions of the Model}

This section discusses several extensions of our basic model. Our main purpose in this exercise is to emphasize that even with these added features the basic trade-offs we have identified in this paper remain. Given this purpose, much of the discussion is kept deliberately informal. We also focus in all cases below on comparing the benchmark reincentivization and pre-commitment strategies, since the intuitive arguments are clearest in this context.

We discuss four extensions here. Sections 7.1 and 7.2 allow for a termination of the principalmanager relationship at the interim information point initiated by, respectively, the principal (Section 7.1) and the manager (Section 7.2). Section 7.3 then looks at the optimality of resetting as a

\footnotetext{
${ }^{17}$ Although the table reports values only upto $e=0.50, \hat{\beta}_{e}$ remains strictly positive even as $e$ gets close to its upper-bound of unity. For example, for $e=0.90$, the optimal levels of $\hat{\beta}_{e}$ in the three panels are, respectively, 0.181, 0.209 , and 0.219 .
} 


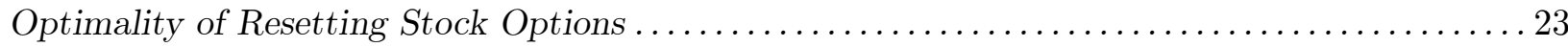

function of the relative control the manager has over the returns distribution. Lastly, Section 7.4 explains why that our results would be essentially unaffected by allowing more general compensation structures.

\subsection{Allowing Dismissal of the Manager}

Our model presumes the principal/manager relationship lasts the full length of the horizon, in particular, that neither party may elect to terminate the relationship at $t=1$. This is not an unreasonable assumption to make since our model is, in effect, a one-period model with an interim time point where information is received. Nonetheless, it seems worthwhile to examine the consequences of allowing a termination of the relationship, subsequent to the revelation of information. In this subsection, we examine the impact of allowing the principal to fire the manager at time 1; the subsection immediately following looks at the case where the manager can quit at this interim point. We argue in either case that, under reasonable assumptions, the addition of these features complicates our model notationally, but does not add anything essential to the incentivization-vs-feedback issue at the heart of this paper.

So suppose the principal can dismiss the incumbent manager after one period. We first analyze the case where the dismissed manager is replaced with an ex-ante identical manager under the assumption that this replacement is costless for the principal. Under this condition, we will show that the pre-commitment and resetting strategies are equivalent for the principal.

Take any strategy for the principal that uses resetting. Consider the pre-commitment strategy which makes the same initial offer and proceeds as follows: at any node where the resetting strategy fires the manager, the pre-commitment strategy also fires the manager; and at any node where the resetting strategy alters the terms of an existing contract, the pre-commitment strategy fires the manager, and offers the new manager the reset contract. Then, the continuation payoffs for the principal from any node are never worse under pre-commitment than under resetting. Moreover, the initial incentives are evidently no weaker under pre-commitment. It is immediate that precommitment cannot do worse than resetting.

Conversely, take any strategy under pre-commitment. Consider the strategy under resetting which makes the same initial offer and proceeds as follows. The strategy fires the manager at any node where either (a) the pre-commitment strategy fires the manager, or (b) a Pareto-improving resetting is possible, but would have a negative feedback effect. (If resetting would also have a positive feedback effect, the strategy does not fire and resets accordingly.) The initial incentives are then no weaker under resetting than under pre-commitment, and the continuation payoffs are never worse for the principal. Therefore, resetting must do at least as well as pre-commitment. Thus, the two must be equivalent in equilibrium.

This equivalence is less impressive than it appears at first blush. It is driven by the assumption that the manager may be replaced in mid-stream at no cost. In effect, this assumption presents the principal with a situation in which superior continuation payoff patterns can be generated without weakening initial incentives through a feedback effect. In practice, however, many costs exist which may drive a wedge between the continuation values that may be realized using incumbent managers and those obtainable by replacement. (The presence of such costs would also explain what would otherwise be irrational: why corporations may prefer to retain and reincentivize existing managers 
than to replace them.) There may be explicit costs such as hiring costs or costs associated with terminating the original contract. There may be implicit costs (i.e., productivity losses) such as the presence of learning-by-doing effects on account of which the new manager will not initially be as productive as the one replaced. There may be adverse selection considerations on account of which untried managers may have uncertain productivity characteristics.

When such costs exist, the optimal resetting of an existing contract will result in a strictly higher continuation payoff than replacing the manager and offering the new manager an optimal contract for the continuation; and, of course, the "tighter" the human resource constraint (i.e., the higher the costs of replacement), the greater will be this difference. Thus, allowing for resetting provides for superior continuations, but - via the feedback effect-has a negative effect on initial incentives. Conversely, pre-committing to firing the manager at certain nodes will provide superior initial incentives but poor continuation payoffs at those nodes. These trade-offs are precisely the ones at the heart of the current paper.

\subsection{Allowing the Manager to Quit}

Corporations that have reset options have typically offered two reasons justifying their actions. One is the need to reincentivize employees who have become demotivated because of their underwater options; this point has, of course, formed the focus of our paper. A second reason is also commonly offered for resetting: that it serves as a device to help retain employees who might otherwise quit. If our model is extended to allow for the manager to resign after one period, it can be shown that the same results as in Section 7.1 hold: (a) if there are no implicit or explicit costs of replacing the incumbent manager, pre-commitment and resetting are equivalent strategies; and (b) this equivalence is broken, and the choice between these alternatives resembles the issues discussed in this paper, if more reasonable assumptions are made concerning these costs. The details are in our working paper; we omit them here.

\subsection{Managerial Control and the Optimality of Resetting}

Resetting stock options sometimes is defended by suggesting that the poor performance preceding the resetting was driven by factors beyond the manager's control. In this section, we examine the extent to which such an argument may be justified.

As the first step in this process, we generalize the linear-quadratic model of Section 4.2 to incorporate a notion of managerial "control" over return generation. Specifically, we assume that given an action $a \in[0, \bar{a}]$ for the manager, we have

$$
\operatorname{Prob}(H)=q \mu+(1-q) a=1-\operatorname{Prob}(L)
$$

where $\mu$ and $q$ are given parameters in [0,1]. The expression (7.1) and the interpretation of $\mu$ and $q$ may be motivated in the following way. Suppose that when the action $a$ is taken, the outcomes are realized independently of the manager's action with probability $q$, with the likelihood of the different outcomes given by

$$
\operatorname{Prob}(H)=\mu \quad \operatorname{Prob}(L)=1-\mu,
$$


Optimality of Resetting Stock Options ..................................... 25

and that with probability $1-q$, the outcome distribution is determined by $a$, with

$$
\operatorname{Prob}(H)=a \quad \operatorname{Prob}(L)=1-a .
$$

Then, the parameter $q$ has an obvious interpretation as a measure of managerial control: ceteris paribus, the lower is $q$, the higher is the influence of the manager's action in determining the likelihoods of final rewards. The parameter $\mu$ determines the direction of impact of the external factors. Other things being equal, a higher value for $\mu$ implies a higher overall likelihood of $H$. As such, high values of $\mu$ connote favorable outside factors (such as higher industry or economywide growth) that enhance firm performance, while low values of $\mu$ indicate unfavorable external conditions (such as recessionary trends in the market). Moreover, combining (7.2) and (7.3), the overall probabilities of $H$ and $L$ arise as in (7.1). ${ }^{18,19}$

The specification (7.1) is also particularly advantageous from an analytical standpoint. When the cost structure is quadratic, equilibria under (7.1) may be identified by essentially retracing the steps in Appendix A with a few marginal changes. We omit the details here, focussing instead on the properties of equilibrium under pre-commitment and reincentivization. Table 4 summarizes equilibrium outcomes in two parts. The first panel in the table describes equilibrium payoffs and compensation levels under either regime for a given value of $\mu$ and a range of values of $q$. The second panel in the table presents the difference $V_{R}-V_{C}$ between the principal's values under reincentivization and pre-commitment for a range of values of $\mu$ and $q$.

Consider first the impact of a change in $q$. A striking property that holds in the first panel - and also for other values of $\mu$ that we examined - is that for each fixed $\mu$, the equilibrium values $V_{R}$ and $V_{C}$ to the principal under the two regimes each decrease monotonically as $q$ increases. (This was true even for very high values of $\mu$ (e.g., $\mu=0.95$ ) so the effect of the external factors is positive with a very high likelihood.) Second, of greater interest from the point of view of this paper, the second panel shows the difference $\left(V_{R}-V_{C}\right)$, which measures the relative optimality of resetting, also declines monotonically as $q$ increases.

Both features have a common explanation. As a measure of the influence of factors the manager cannot directly control, $q$ measures, in a sense, the "background risk" faced by the manager. An increase in the level of this risk makes it more difficult for the principal to appropriately incentivize the manager. Thus, for example, in the upper panel of Table 4, the number of options awarded the agent, both initially and at reset time (under reincentivization), increases with $q$. On the one hand, this makes resetting less profitable in the continuation; on the other, the greater continuation awards also carry a potentially larger feedback effect. On both counts, the relative optimality of resetting declines as $q$ increases.

Turning now to behavior in $\mu$, the second panel in Table 4 again exhibits a strong monotonicity property: the difference $\left(V_{R}-V_{C}\right)$ declines as $\mu$ increases, indicating that resetting becomes less relatively optimal as the impact of external factors becomes more positive. (It is also true, though the table does not show this, that for each fixed value of $q, V_{R}$ and $V_{C}$ are both increasing in $\mu$.)

\footnotetext{
${ }^{18}$ We stress that these probabilities are confounding: the principal only observes the outcome $H$ or $L$, and cannot determine whether the outcome was influenced by external factors or the manager's actions.

${ }^{19}$ We note that the impact of external factors remains qualitatively identical if we replace the "convex combination" specification (7.1) with $\operatorname{Prob}(H)=m+\xi a$, where $m$ and $\xi$ are positive quantities denoting, respectively, the contributions of the external factor and the manager. The details are available from the authors.
} 


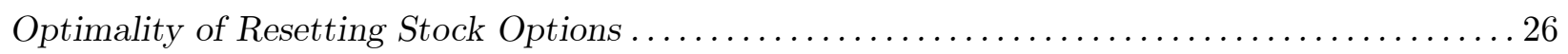

Table 4: The Effect of Background Risk on Equilibrium Outcomes

This table describes the structure of equilibrium payoffs and actions under both pre-commitment and resetting under the specifications of Section 7.3. The base parameters are fixed at $H=1.20$, $L=0.833$, and $\bar{a}=0.9$. The parameters $\mu$ and $q$ denote, respectively, the directional impact of background noise, and the measure of managerial influence to background noise in generating outcomes. The cost parameter is taken to be $k=0.02$. The subscripts $R$ and $C$ refer to "reincentivization" and "pre-commitment," respectively; $V$ and $U$ are, respectively, the principal's and manager's expected utilities in equilibrium; $\alpha$ is the size of initial option award; and $\beta$ is the size of the reset award under reincentivization. The first panel in the table considers equilibrium outcomes when $\mu=0.65$ for a range of values of $q$. The second panel presents the difference $\left(V_{R}-V_{C}\right)$ for ranges of values of both $\mu$ and $q$.

\begin{tabular}{||c||c|c|c|c|c|c|c||}
\hline \multicolumn{7}{||c||}{ Equilibrium Outcomes: $\mu=0.65$} \\
\hline$q$ & 0.350 & 0.400 & 0.450 & 0.500 & 0.550 & 0.600 & 0.650 \\
\hline$V_{R}$ & 1.280 & 1.262 & 1.243 & 1.224 & 1.204 & 1.182 & 1.158 \\
$V_{C}$ & 1.274 & 1.256 & 1.239 & 1.222 & 1.205 & 1.188 & 1.171 \\
\hline$U_{R}$ & 0.034 & 0.037 & 0.041 & 0.046 & 0.052 & 0.059 & 0.068 \\
$U_{C}$ & 0.017 & 0.019 & 0.021 & 0.023 & 0.026 & 0.030 & 0.034 \\
\hline$\alpha_{R}$ & 0.150 & 0.164 & 0.181 & 0.201 & 0.226 & 0.257 & 0.298 \\
$\alpha_{C}$ & 0.106 & 0.115 & 0.125 & 0.138 & 0.153 & 0.173 & 0.198 \\
\hline$\beta$ & 0.185 & 0.200 & 0.218 & 0.240 & 0.267 & 0.300 & 0.343 \\
\hline
\end{tabular}

\begin{tabular}{||c||c|c|c|c|c|c|c||}
\hline \multicolumn{1}{||c|}{$V_{R}-V_{C}$} \\
\hline$q \backslash \mu$ & 0.350 & 0.450 & 0.550 & 0.650 & 0.750 & 0.850 & 0.950 \\
\hline 0.35 & 0.031 & 0.023 & 0.015 & 0.006 & -0.002 & -0.009 & -0.017 \\
0.40 & 0.031 & 0.023 & 0.015 & 0.006 & -0.003 & -0.011 & -0.019 \\
0.45 & 0.031 & 0.022 & 0.013 & 0.004 & -0.004 & -0.013 & -0.022 \\
0.50 & 0.029 & 0.020 & 0.011 & 0.002 & -0.007 & -0.016 & -0.025 \\
0.55 & 0.027 & 0.018 & 0.008 & -0.001 & -0.011 & -0.020 & -0.030 \\
0.60 & 0.023 & 0.014 & 0.004 & -0.006 & -0.016 & -0.025 & -0.035 \\
0.65 & 0.018 & 0.008 & -0.002 & -0.012 & -0.022 & -0.032 & -0.042 \\
\hline
\end{tabular}

These are intuitive properties. Ceteris paribus, as $\mu$ increases, resetting is less profitable in the continuation, since the manager's incentive to work is reduced at the margin; the feedback effect is also larger as the manager benefits from the added positive impact of the external noise. Thus, pre-commitment strategies become relative more attractive.

\subsection{More General Compensation Structures}

The analysis in this paper has been conducted on the basis that the only compensation instruments used by the principal are (analogs of) at-the-money call options on the firm's terminal equity value. This condition may appear restrictive, but it is conceptually quite unimportant. We elaborate on this below.

The central point underlying our analysis has been that allowing for interim renegotiation of contract terms will, in general, create greater sensitivity to observed histories - or path-dependence - in 
final payoffs, but will also have the effect of weakening initial incentives. Focussing on options as the sole instruments of compensation enabled us to capture the tension between these forces in a particularly stark manner. However, adding other commonly-used methods of compensation would not have changed the substance of our results. Consider equity, for example. Under precommitment, any initial award of equity has a final value that is independent of the path taken to reach that value; in our model, for instance, the value of any initial award of equity is the same at the final nodes $H L$ and $L H$. On the other hand, allowing for interim rewriting of the contract terms (in particular, allowing the size of the grant to be altered), introduces greater flexibility into the final payoffs; of course, to the extent it is anticipated, it also weakens initial incentives. This is exactly the trade-off we have studied in this paper.

Indeed, it is apparent that the only general case in which this trade-off is irrelevant is one where resetting fails to expand the set of potential final payoff profiles, i.e., one in which the principal already has the ability to write contracts of arbitrary complexity that could be contingent on interim events in fine ways. In such a situation, of course, pre-commitment can never be worse than resetting: one available alternative for the principal is to commit to the payoff profile that would be realized under resetting, thereby creating the same initial and interim incentives for the manager as resetting. The converse, however, is false. Under pre-commitment, the principal can commit to inefficient continuations from some interim states (in the interests of generating appropriate initial incentives), but under resetting such an offer fails to be credible, since the manager will correctly anticipate that the contract terms will be amended at the relevant nodes.

We do not find a case in which the principal has such unrestricted contracting ability compelling. It is well known that in such circumstances, theoretically optimal contracts will typically depend on observed histories in very fine and complex ways. In contrast, long-term compensation instruments used in practice (such as options) overwhelmingly tend to be path-independent; indeed, it is this very feature that necessitates resetting to realign incentives. A second consideration that also argues against allowing for unlimited contracting ability (pointed out by this paper's referee), is simply that contracting is a costly procedure. It is too expensive to anticipate and initially contract about all conceivable future states. All of this suggests that it is true but irrelevant that with instruments of unlimited complexity, shareholder value cannot be enhanced by resetting contracts at any point. However, it is both relevant and of interest to ask - as we have done in this paper - whether, given that instruments of limited complexity are used to set compensation, it could be optimal to agree to amend the terms of the original contract in some contingencies.

\section{Conclusion}

It has become commonplace in recent years for firms which have experienced a decline in share prices to reduce the strike price on previously-awarded stock option grants in a bid to bring them closer to the money. Reaction to this practice in the financial press and from large institutional investors has been almost wholly negative. Yet, surprisingly, the possible optimality and incentive effects of "resetting" have not been addressed in a contracting model in the literature on executive compensation. This paper describes a model for this purpose.

We find that although the anticipation of resetting does weaken incentives present in the original 
award (the most common criticism of this practice), resetting can nonetheless be a value-enhancing strategy for firms to employ, even in an ex-ante sense. In a comparison of natural benchmarks, we find that under robust conditions, the strategy that pre-commits to no resetting could be strictly dominated by the polar opposite strategy of reincentivization which resets optimally for the continuation whenever this is profitable. Using a more general class of strategies, we show, moreover, that some degree of resetting is always optimal in all cases. This remains true even if managers can adversely influence the resetting process and cause "too much" resetting to occur, except when a combination of restrictive circumstances holds. In summary, these results indicate that the case against resetting is quite weak.

We also show that the nature of the trade-offs we study is essentially unchanged if we extend the model to allow either party to sever the relationship in the interim. Finally, we also examine a commonly-cited defense of resetting as necessitated by a decline in share prices due to "factors beyond the manager's control." We find that although resetting may continue to be important even when external factors play a role in determining the return distribution, it becomes relatively less optimal as the role of the manager diminishes relative to these factors. In particular, resetting is most likely to be important when the manager's actions have a large impact on final outcomes. This last point offers one possible rationalization of the greater frequency of resetting empirically documented among smaller firms.

Finally, we note the framework we develop here has analogies to a number of other settings in financial economics as well. One of particular interest is the design of bankruptcy procedures. On the one hand, bankruptcy systems which encourage renegotiation between the concerned parties (as in the US) are akin to the situation where the option is reset contingent on a bad outcome being realized. Such systems have the advantage that continuation values are efficiently realized, but they have an evident feedback effect that could increase the probabilities of bankruptcies occurring. On the other hand, insolvency codes which effectively precipitate liquidation of the firm under bankruptcy (as in Germany) are analogous to pre-commitment strategies that eliminate renegotiation possibilities or, at least, make them very difficult. This leads to potentially inefficient continuations, but may have a beneficial feedback effect that lowers the occurrence of bankruptcies. 


\section{A Equilibrium in the Model of Section 5.2}

This section derives equilibria in the linear/quadratic model of Section 4.2. Section A.1 looks at the pre-commitment game, while Section A.2 describes how equilibria may be identified when resetting is permitted.

\section{A.1 Equilibrium under Pre-commitment}

Let $\alpha$ be the number of call options (each with a strike of unity) awarded the agent at time- 0 . Contingent upon reaching the node $H$, the agent solves

$$
\max _{a \in[0, \bar{a}]}\left\{a \cdot \alpha\left(H^{2}-1\right)+(1-a) \cdot 0-\frac{1}{2} k a^{2}\right\}
$$

This has the solution

$$
a_{h}(\alpha)= \begin{cases}\alpha\left(H^{2}-1\right) / k, & \text { if } \alpha<\bar{a} k /\left(H^{2}-1\right) \\ \bar{a}, & \text { otherwise }\end{cases}
$$

Thus, the agent's and principal's continuation utilities $U_{h}(\alpha)$ and $V_{h}(\alpha)$ from the node $H$ are given by

$$
\begin{aligned}
& U_{h}(\alpha)=a_{h}(\alpha) \alpha\left(H^{2}-1\right)-\frac{1}{2} k\left[a_{h}(\alpha)\right]^{2} . \\
& V_{h}(\alpha)=a_{h}(\alpha)\left[H^{2}-\alpha\left(H^{2}-1\right)\right]+\left(1-a_{h}(\alpha)\right) H L .
\end{aligned}
$$

At node $L$, the options are guaranteed to finish out of the money, so we trivially have $a_{l}=U_{l}=0$ and $V_{l}=L^{2}$. At the initial node, now, the agent solves

$$
\max _{a \in[0, \bar{a}]}\left[a U_{h}(\alpha)+(1-a) U_{l}-\frac{1}{2} k a^{2}\right]
$$

Using $U_{l}=0$, the optimal first-period action for the agent is

$$
a(\alpha)= \begin{cases}U_{h}(\alpha) / k, & \text { if } U_{h}(\alpha) \leq \bar{a} k \\ \bar{a}, & \text { otherwise }\end{cases}
$$

Taking into account the agent's optimal response (A.1)-(A.4) to an initial offer of $\alpha$, the principal now picks $\alpha$ to maximize his initial expected utility:

$$
\max _{\alpha \geq 0}\left[a(\alpha) V_{h}(\alpha)+(1-a(\alpha)) V_{l}\right]
$$


Our goal is to identify the value of $\alpha$ that solves (A.5). For notational simplicity, we will suppress dependence on $\alpha$ in the sequel. There are four possibilities that could be induced by $\alpha$ in equilibrium: (i) $a_{h}<\bar{a}, a<\bar{a}$; (ii) $a_{h}<\bar{a}, a=\bar{a}$; (iii) $a_{h}=\bar{a}, a<\bar{a}$; and (iv) $a_{h}=a=\bar{a}$. We look at each of these in turn to see if such equilibria exist.

Case $1 a_{h}<\bar{a}, a<\bar{a}$.

The inequalities $a_{h}<\bar{a}$ and $a<\bar{a}$ can hold only if $a_{h}=\alpha\left(H^{2}-1\right) / k$ and $a=U_{h} / k$. Of course, for these to hold, $\alpha$ must, in turn, satisfy $\alpha<\bar{a} k /\left(H^{2}-1\right)$ and $U_{h}(\alpha)<\bar{a} k$. We will assume for the moment that $\alpha$ satisfies these conditions, solve for the optimal value of $\alpha$, and see whether our assumptions were justified at this optimal value. If so, the solution qualifies as a candidate equilibrium initial offer; if not, equilibria of this form evidently do not exist.

Under the presumed hypotheses, we have $U_{h}=\alpha^{2}\left(H^{2}-1\right)^{2} / 2 k$ and $V_{h}=H L+\left[\alpha\left(H^{2}-1\right)\left(H^{2}-\right.\right.$ $\left.H L)-\alpha^{2}\left(H^{2}-1\right)^{2}\right] / k$. Substituting these expressions into (A.5) and maximizing with respect to $\alpha$ results in a quadratic in $\alpha\left(H^{2}-1\right)$. Solving this quadratic yields

$$
\alpha=\frac{-m+\sqrt{m^{2}-4 l n}}{2 l}
$$

where

$$
\begin{aligned}
l & =2\left(H^{2}-1\right)^{2} / k \\
m & =-3\left(H^{2}-1\right)\left(H^{2}-H L\right) / 2 k \\
n & =-\left(H L-L^{2}\right)
\end{aligned}
$$

Expression (A.6) is retained as a candidate equilibrium solution is it satisfies the two assumptions under which it was derived. (This is easy to check for any given specific values of the parameters.) If either inequality is violated, then no equilibria of the desired form exist under the chosen parameters.

Case $2 a_{h}<\bar{a}, a=\bar{a}$.

A simple set of computations using (A.1)-(A.4) shows that these inequalities can hold together only if $\bar{a}>2$. Since this is impossible, we discard this case.

Case $3 a_{h}=\bar{a}, a<\bar{a}$.

In this case, the continuation utilities from $H$ are given by

$$
\begin{aligned}
& U_{h}=\bar{a} \alpha\left(H^{2}-1\right)-\frac{1}{2} k \bar{a}^{2} . \\
& V_{h}=\bar{a}\left[H^{2}-\alpha\left(H^{2}-1\right)\right]+(1-\bar{a}) H L .
\end{aligned}
$$


Thus, the agent chooses $a$ to solve

$$
\max _{a \in[0, \bar{a}]}\left\{a\left[\bar{a} \alpha\left(H^{2}-1\right)-\frac{1}{2} k \bar{a}^{2}\right]-\frac{1}{2} k a^{2}\right\}
$$

Since we are assuming the interior solution for $a$, we have $a=U_{h} / k$. Using this, we seek the value of $\alpha$ that maximizes (A.5). The solution is easily seen to be

$$
\alpha=\frac{1}{2 \bar{a}\left(H^{2}-1\right)}\left[\bar{a} H^{2}+(1-\bar{a}) H L-L^{2}+\frac{1}{2} k \bar{a}^{2}\right] .
$$

Once again, this solution is discarded if it violates either of the presumed hypotheses that $\alpha>$ $k \bar{a} /\left(H^{2}-1\right)$ or $U_{h} \leq \bar{a} k$.

Case $4 a_{h}=\bar{a}, a=\bar{a}$.

Now, we must have $\alpha \geq \bar{a} k /\left(H^{2}-1\right)$ and $U_{h} \geq \bar{a} k$. It is apparent that, in this case, it is in the principal's interest to choose the smallest value of $\alpha$ that guarantees these outcomes; this value may be seen to be

$$
\alpha=\frac{k(2+\bar{a})}{2\left(H^{2}-1\right)}
$$

Having identified potential solutions of all four forms, it is now a trivial matter, given any set of values for the parameters $H, L, \bar{a}$ and $k$, to identify the set of candidate solutions. Comparison of the value of the objective function at these solutions (and at the point $\alpha=0$ ) establishes the optimal value of $\alpha$ under pre-commitment.

\section{A.2 Equilibrium under Reincentivization}

When reincentivization is possible, equilibria are a little harder to identify because of the possibility of the constrained resetting at $H$ and $L$. We proceed in three steps. First, we identify equilibrium resetting at $L$. Then, we identify the problem whose solution yields the equilibrium initial offer $\alpha$ in this problem, taking into account the possibility of resetting at both $H$ and $L$. Finally, we discuss deriving the solution to this problem.

\section{Step 1: Equilibrium Resetting at $L$}

For any value of the initial offer $\alpha$, the initial options are worthless at $L$. Thus, if no resetting of the initial contract is made, the agent's optimal response in the continuation is $a_{l}=U_{l}=0$, which 
gives the principal a continuation utility of $V_{l}=L^{2}$. However, if a new award of $\beta$ at-the-money options is made, then the agent solves

$$
\max _{a \in[0, \bar{a}]}\left\{a \beta(H L-L)-\frac{1}{2} k a^{2}\right\}
$$

This has the solution $a_{l}(\beta)=\min \{\bar{a}, \beta(H L-L) / k\}$. The equilibrium reset contract is the one which maximizes the principal's continuation utility from $L$ :

$$
\max _{\beta \in[0,1]}\left\{a_{l}(\beta)(H L-\beta(H L-L))+\left(1-a_{l}(\beta)\right) L^{2}\right\}
$$

Some computation shows that the solution to this problem is given by ${ }^{20}$

$$
\beta= \begin{cases}(H-L) /[2(H-1)], & \text { if } L(H-L) \leq 2 \bar{a} k \\ k \bar{a} /(H L-L), & \text { otherwise }\end{cases}
$$

As a consequence, the continuation values $U_{l}$ and $V_{l}$ for the principal and agent are given by

$$
\begin{aligned}
& U_{l}= \begin{cases}L^{2}(H-L)^{2} / 8 k, & \text { if } L(H-L) \leq 2 \bar{a} k \\
k \bar{a}^{2} / 2, & \text { otherwise }\end{cases} \\
& V_{l}= \begin{cases}L^{2}+L^{2}(H-L)^{2} / 4 k, & \text { if } L(H-L) \leq 2 \bar{a} k \\
\bar{a} H L+(1-\bar{a}) L^{2}-k \bar{a}^{2}, & \text { otherwise }\end{cases}
\end{aligned}
$$

Note that in the first case (when $L(H-L) \leq 2 \bar{a} k$ ), it is clearly optimal to reset the contract at $L$ since the continuation value is strictly larger than $L^{2}$; this also holds in the second case provided $L(H-L)>k \bar{a}$. In the sequel, we continue with only the first case. The second is easily handled analogously.

\section{Step 2: The Equilibrium Initial Offer $\alpha^{*}$}

Pick any value of $\alpha$ and define the continuation action $a_{h}(\alpha)$ and the continuation values $U_{h}(\alpha)$ and $V_{h}(\alpha)$ as in (A.1)-(A.3). With $U_{l}$ given by (A.11), let $a(\alpha)$ be the solution to

$$
\max _{a \in[0, \bar{a}]}\left\{a U_{h}(\alpha)+(1-a) U_{l}-\frac{1}{2} k a^{2}\right\}
$$

Finally, with $V_{l}$ given by (A.12), let $\alpha^{*}$ be the optimum value of $\alpha$ in the problem

$$
\max _{\alpha \in[0,1]}\left\{a(\alpha) V_{h}(\alpha)+(1-a(\alpha)) V_{l}\right\} .
$$

\footnotetext{
${ }^{20}$ We must also ensure that $\beta \leq 1$. This is satisfied whenever $\bar{a} k \leq(H L-L)$, an assumption we shall make here and in our numerical calculations in Table 2.
} 
In words, $\alpha^{*}$ is the optimal initial offer to make for the principal if resetting will occur at $L$ but not at $H$. Of course, the principal cannot commit himself a priori to not resetting at $H$. Nonetheless, we will show that (i) $\alpha^{*}$ is an optimal initial offer to make under resetting, and (ii) if $a\left(\alpha^{*}\right)>0$, then there will be no resetting at the node $H$ under the offer $\alpha^{*}$. The following lemma will help establish these claims:

Lemma A.1 In any solution to (A.14), it is the case that either $a\left(\alpha^{*}\right)=0$ or $V_{h}\left(\alpha^{*}\right) \geq V_{l}$.

Proof Suppose we had $a\left(\alpha^{*}\right)>0$ and $V_{h}\left(\alpha^{*}\right)<V_{l}$. Then, of course, we must have

$$
V_{l}>a\left(\alpha^{*}\right) V_{h}\left(\alpha^{*}\right)+\left(1-a\left(\alpha^{*}\right)\right) V_{l} .
$$

Now, the principal can always achieve the value $V_{l}$ in (A.14) by using $\alpha=0$, since this guarantees the agent's first-period action will be $a=0$. Together with (A.15), this implies $\alpha^{*}$ cannot maximize (A.14), a contradiction.

We return to the proof of the claim. We will first show that if the initial offer $\alpha^{*}$ is made and $a\left(\alpha^{*}\right)>0$, then it cannot be optimal to reset at $H$. Suppose to the contrary that under these conditions, a Pareto-improving continuation exists from $H$ that leaves the principal strictly better off. For specificity, suppose this continuation involves issuing $\gamma$ new options each with a strike of $H$ to replace the existing $\alpha^{*}$ options. We will show that this means $\alpha^{*}$ does not solve (A.14), a contradiction.

Let $U_{h}(\gamma)$ and $V_{h}(\gamma)$ represent the continuation payoffs from $H$ under $\gamma$. By hypothesis, $U_{h}(\gamma) \geq$ $U_{h}\left(\alpha^{*}\right)$ and $V_{h}(\gamma)>V_{h}\left(\alpha^{*}\right)$. Now consider the effect of setting $\alpha=\gamma H /(H+1)$ in (A.14). Under $\alpha$, the payoffs to the agent and the principal at the nodes $H^{2}$ and $H L$ are the same as under $\gamma$; therefore, we must have $U_{h}(\alpha)=U_{h}(\gamma)$ and $V_{h}(\alpha)=V_{h}(\gamma)$, and this implies in turn

$$
U_{h}(\alpha) \geq U_{h}\left(\alpha^{*}\right), \quad V_{h}(\alpha)>V_{h}\left(\alpha^{*}\right)
$$

The first inequality states that the offer $\alpha$ does not decrease the "spread" $\left(U_{h}-U_{l}\right)$, and so from (A.13) the action $a(\alpha)$ it induces must satisfy $a(\alpha) \geq a\left(\alpha^{*}\right)$. Combining this with the second inequality and the inequality $V_{h}\left(\alpha^{*}\right) \geq V_{l}$ (which must hold by Lemma A.1), we have

$$
a(\alpha) V_{h}(\alpha)+(1-a(\alpha)) V_{l}>a\left(\alpha^{*}\right) V_{h}\left(\alpha^{*}\right)+\left(1-a\left(\alpha^{*}\right)\right) V_{l} .
$$

But this means $\alpha$ does better than $\alpha^{*}$ in (A.14), a contradiction. This completes the proof that resetting cannot be optimal at $H$ if the initial offer is $\alpha^{*}$.

Finally, suppose that $\alpha^{*}$ is not an optimal initial offer under resetting; specifically, suppose there is another initial offer $\hat{\alpha}$ that does strictly better. Then, $\hat{\alpha}$ must involve resetting at $H$; if it did not, it could not do better than $\alpha^{*}$, since $\alpha^{*}$ solves (A.14). Let the resetting at $H$ be $\gamma$ at-the-money options. Define $U_{h}(\gamma)$ and $V_{h}(\gamma)$ as earlier, and denote by $a(\gamma)$ the first period action induced by 
$\gamma$. Note that the initial expected utility of the principal under $\hat{\alpha}$ is then $\left[a(\gamma) V_{h}(\gamma)+(1-a(\gamma)) V_{l}\right]$, which, by hypothesis, is strictly larger than $a\left(\alpha^{*}\right) V_{h}\left(\alpha^{*}\right)+\left(1-a\left(\alpha^{*}\right)\right) V_{l}$.

Consider now setting $\alpha=\gamma H /(H+1)$ in (A.14). This offer induces the same endgame payoffs as $\gamma$ at the nodes $H^{2}$ and $H L$, so we clearly have $U_{h}(\alpha)=U_{h}(\gamma)$ and $V_{h}(\alpha)=V_{h}(\gamma)$; and, as a consequence, $a(\alpha)=a(\gamma)$. Summing up:

$$
a(\alpha) V_{h}(\alpha)+(1-a(\alpha)) V_{l}=a(\gamma) V_{h}(\gamma)+(1-a(\gamma)) V_{l}
$$

But this means $\alpha$ does better than $\alpha^{*}$ in (A.14), contradicting the definition of the latter. This establishes the claim.

\section{Step 3: Solving for the Optimal Initial Offer $\alpha^{*}$}

To solve for $\alpha^{*}$, we must solve (A.14). This is completely analogous to the procedure for solving for the pre-commitment equilibrium in (A.5). There are again four cases to consider: where $\alpha^{*}$ induces (i) $a_{h}<\bar{a}, a<\bar{a}$; (ii) $a_{h}<\bar{a}, a=\bar{a}$; (iii) $a_{h}=\bar{a}, a<\bar{a}$; and (iv) $a_{h}=a=\bar{a}$. The derivation of potential equilibria of each sort may be carried out as earlier. For example, it may be verified that if an equilibrium $\alpha^{*}$ exists which induces $a_{h}<\bar{a}$ and $a<\bar{a}$, then $\alpha^{*}$ must satisfy the equation

$$
\left(V_{h}-V_{l}\right) \lambda^{2} \alpha\left(H^{2}-1\right)^{2}+a\left[\lambda\left(H^{2}-1\right)\left(H^{2}-H L\right)-2 \lambda \alpha\left(H^{2}-1\right)^{2}\right]=0 .
$$

(Once again, of course, to qualify as a candidate solution, it must be verified that $\alpha^{*}$ so defined meets the assumptions under which it was derived.) Proceeding as we did earlier, we identify all the possible solutions. Comparison of the value of the objective function at all feasible candidate solutions then delivers the equilibrium. 
Optimality of Resetting Stock Options

\section{References}

[1] APB Opinion No. 25, "Accounting for Stock Issued to Employees," Accounting Principles Board, 1972.

[2] Aghion, P. and P. Bolton (1992) An Incomplete Contracts Approach to Financial Contracting, Review of Economic Studies 59, 473-494.

[3] Aghion, P., M. Dewatripont, and P. Rey (1990) On Renegotiation Design, European Economic Review 34, 322-329.

[4] Bolton, P. (1990) Renegotiation and the Dynamics of Contract Design, European Economic Review 34, 303-310.

[5] Bolton, P. and D. Scharfstein (1989) A Theory of Predation Based on Agency Problems in Financial Contracting, American Economic Review 80, 94-106.

[6] Bolton, P. and D. Scharfstein (1996) Optimal Debt Structure and the Number of Creditors, Journal of Political Economy 104(1), 1-25.

[7] Brenner, M., R. Sundaram, and D. Yermack (1998) Altering the Terms of Executive Stock Options, Working Paper, Department of Finance, Stern School of Business; forthcoming, Journal of Financial Economics.

[8] Chance, D., R. Kumar, and R. Todd (1997) The "Repricing" of Executive Stock Options, mimeo, Department of Finance, Virginia Tech.

[9] Franks, J., K. Nyborg, and W. Torous (1996) A Comparison of US, UK, and German Insolvency Codes, Financial Management 25(3), 86-101.

[10] Gale, D. and M. Hellwig (1989) Repudiation and Renegotiation: The Case of Sovereign Debt, International Economic Review 30(1).

[11] Gilson, S. and M. Vetsuypens (1993) CEO Compensation in Financially Distressed Firms: An Empirical Analysis, Journal of Finance 48, 425-458.

[12] Gorton, G. and B. Grundy (1997) Executive Compensation and the Optimality of Managerial Entrenchment, Working Paper, Wharton School, University of Pennsylvania.

[13] Hart, O. and J. Moore (1988) Incomplete Contracts and Renegotiation, Econometrica 56(4), $755-785$.

[14] Hart, O. and J. Moore (1994) A Theory of Debt based on the Inalienability of Human Capital, Quarterly Journal of Economics 109, 841-880.

[15] Hart, O. and J. Moore (1995) Debt and Seniority: An Analysis of the Role of Hard Claims in Constraining Management, American Economic Review 85, 567-585.

[16] Hart, O. and J. Moore (1998) Default and Renegotiation: A Dynamic Model of Debt, Quarterly Journal of Economics 113(1), 1-41. 
[17] Hart, O. and J. Tirole (1988) Contract Renegotiation and Coasian Dynamics, Review of Economic Studies 55.

[18] Johnson, S. and Y. Tian (1999) The Value and Incentive Effects of Non-Traditional Executive Stock Option Plans, Working Paper, University of Cincinnati.

[19] Kaiser, K. (1996) European Bankruptcy Laws: Implications for Corporations facing Financial Distress, Financial Management 25(3), 67-85.

[20] Radner, R. (1981) Repeated Principal-Agent Games with Discounting, Econometrica 53, 117397.

[21] Radner, R. (1998) Dynamic Games in Organization Theory, in Organizations with Incomplete Information: Essays in Economic Analysis (M. Majumdar, Ed.), Cambridge University Press, Cambridge and New York.

[22] Rogerson, W. (1985) Repeated Moral Hazard, Econometrica 53, 69-77.

[23] Saly, P.J. (1994) Repricing Executive Stock Options in a Down Market, Journal of Accounting and Economics 18, 325-356.

[24] Statement of Financial Accounting Standards No. 123, "Accounting for Stock-Based Compensation," Financial Accounting Standards Board, October 1995. 